\title{
Classical-Quantum Arbitrarily Varying Wiretap Channel: Secret Message Transmission under Jamming Attacks
}

\author{
Holger Boche \\ Lehrstuhl für Theoretische Informationstechnik, \\ Technische Universität München, \\ Munich, Germany \\ boche@tum.de \\ Minglai Cai \\ Lehrstuhl für Theoretische Informationstechnik, \\ Technische Universität München, \\ Munich, Germany \\ minglai.cai@tum.de \\ Christian Deppe \\ Fakultät für Mathematik, \\ Universität Bielefeld, \\ Bielefeld, Germany \\ cdeppe@mathematik.uni-bielefeld.de \\ Janis Nötzel \\ Universitat Autònoma de Barcelona, \\ Barcelona, Spain/ \\ Technische Universität Dresden \\ Dresden, Germany, \\ Janis.Notzel@uab.cat
}

November 14, 2018

\begin{abstract}
We analyze arbitrarily varying classical-quantum wiretap channels. These channels are subject to two attacks at the same time: one passive (eavesdropping), and one active (jamming). We progress on previous works [11] and [12, by introducing a reduced class of allowed codes that fulfills a more stringent secrecy requirement than earlier definitions. In addition, we prove that non-symmetrizability of the legal link is sufficient for equality of the deterministic and the common randomness assisted secrecy capacities. At last, we focus on analytic properties of both secrecy capacities: We completely characterize their discontinuity points, and their super-activation properties.
\end{abstract}




\section{Contents}

1 Introduction 2

2 Basic Definitions and Communication Scenarios 5

3 Strong Code Concept 12

3.1 Classic Arbitrarily Varying Quantum Wiretap Channel . . . . . . 12

3.2 The Secure Message Transmission With Strong Code Concept . . 19

4 Further Notice on Code Concepts and Applications 26

4.1 Communication With Resources ............. [26

4.2 Some Applications . . . . . . . . . . . . . 33

\section{Introduction}

In the last few years the developments in modern communication systems produced many results in a short amount of time. Especially quantum communication systems allow us to exploit new possibilities while at the same time imposing fundamental limitations.

Quantum mechanics differs significantly from classical mechanics, it has its own laws. Quantum information theory unifies information theory with quantum mechanic, generalizing classical information theory to the quantum world. The unit of quantum information is called the "qubit", the quantum analogue of the classical "bit". Unlike a bit, which is either "0" or "1", a qubit can be in "superposition", i.e. both states at the same time, this is a fundamental tool in quantum information and computing.

A quantum channel is a communication channel which can transmit quantum information. In general, there are two ways to represent a quantum channel with linear algebraic tools, either as a sum of several transformations, or as a single unitary transformation which explicitly includes the unobserved environment.

Quantum channels can transmit both classical and quantum information. We consider the capacity of quantum channels carrying classical information. This is equivalent to considering the capacity of classical-quantum channels, where the classical-quantum channels are quantum channels whose sender's inputs are classical variables. The classical capacity of quantum channels has been determined in [19], [19], 22], and [23].

Our goal is to investigate in communication that takes place over a quantum channel which is, in addition to the noise from the environment, subjected to the action of a jammer which actively manipulates the states. The messages ought also to be kept secret from an eavesdropper.

A classical-quantum channel with a jammer is called an arbitrarily varying classical-quantum channel, where the jammer may change his input in every channel use and is not restricted to use a repetitive probabilistic strategy. In the model of an arbitrarily varying channel, we consider a channel which is not stationary and can change with every use. We interpret this as an attack of a jammer. It works as follows: the sender and the receiver have to select their coding scheme first. After that the jammer makes his choice of the channel state to sabotage the message transmission. However, due to the physical properties, we assume that the jammer's changes only take place in a known set. 
The arbitrarily varying channel was first introduced in [8. 2] showed a surprising result which is known as the Ahlswede Dichotomy: Either the capacity of an arbitrarily varying channel is zero or it equals its shared randomness assisted capacity. After the discovery in [8] it remained as an open question when the deterministic capacity is positive. In 17 a sufficient condition for that has been given, and in [15] it is proved that this condition is also necessary. The Ahlswede Dichotomy demonstrates the importance of shared randomness for communication in a very clear form.

In [4] the capacity of arbitrarily varying classical-quantum channels is analyzed. A lower bound of the capacity has been given. An alternative proof of [4]'s result and a proof of the strong converse are given in [7]. In [3] the Ahlswede Dichotomy for the arbitrarily varying classical-quantum channels is established, and a sufficient and necessary condition for the zero deterministic capacity is given. In [13] a simplification of this condition for the arbitrarily varying classical-quantum channels is given.

In the model of a wiretap channel we consider secure communication. This was first introduced in 27. We interpret the wiretap channel as a channel with an eavesdropper. For a discussion of the relation of the different security criteria we refer to [10] and 24.

A classical-quantum channel with an eavesdropper is called a classical-quantum wiretap channel, its secrecy capacity has been determined in [16] and [14].

This work is a progress of our previous papers [11] and [12, where we considered channel robustness against jamming and at the same time security against eavesdropping. A classical-quantum channel with a jammer and at the same time an eavesdropper is called an arbitrarily varying classical-quantum wiretap channel. It is defined as a family of pairs of indexed channels $\left\{\left(W_{t}, V_{t}\right): t=1, \ldots, T\right\}$ with a common input alphabet and possible different output systems, connecting a sender with two receivers, a legal one and a wiretapper, where $t$ is called a channel state of the channel pair. The legitimate receiver accesses the output of the first part of the pair, i.e., the first channel $W_{t}$ in the pair, and the wiretapper observes the output of the second part, i.e., the second channel $V_{t}$. A channel state $t$, which varies from symbol to symbol in an arbitrary manner, governs both the legal receiver's channel and the wiretap channel. A code for the channel conveys information to the legal receiver such that the wiretapper knows nothing about the transmitted information.

In [11] we established the Ahlswede Dichotomy for arbitrarily varying classicalquantum wiretap channels, i.e., either the deterministic capacity of an arbitrarily varying channel is zero or equals its randomness assisted capacity. Our proof is similar to the proof of the Ahlswede Dichotomy for arbitrarily varying classicalquantum channels in [4]: we build a two-part code word, the first part is used to create the common randomness for the sender and the legal receiver, the second is used to transmit the message to the legal receiver. We also analyzed the secrecy capacity when the sender and the receiver used various resources. In [12] we determined the secrecy capacities under common randomness assisted coding of arbitrarily varying classical-quantum wiretap channels. We also examined when the secrecy capacity was a continuous function of the system parameters. Furthermore, we proved the phenomenon "super-activation" for arbitrarily varying classical-quantum wiretap channels, i.e., there were two channels, both with zero deterministic secrecy capacity, such that if they were used together they allowed perfect secure transmission with positive deterministic secrecy capacity. 
Combining the results of these two paper we get the formula for deterministic secrecy capacity of the arbitrarily varying classical-quantum wiretap channel.

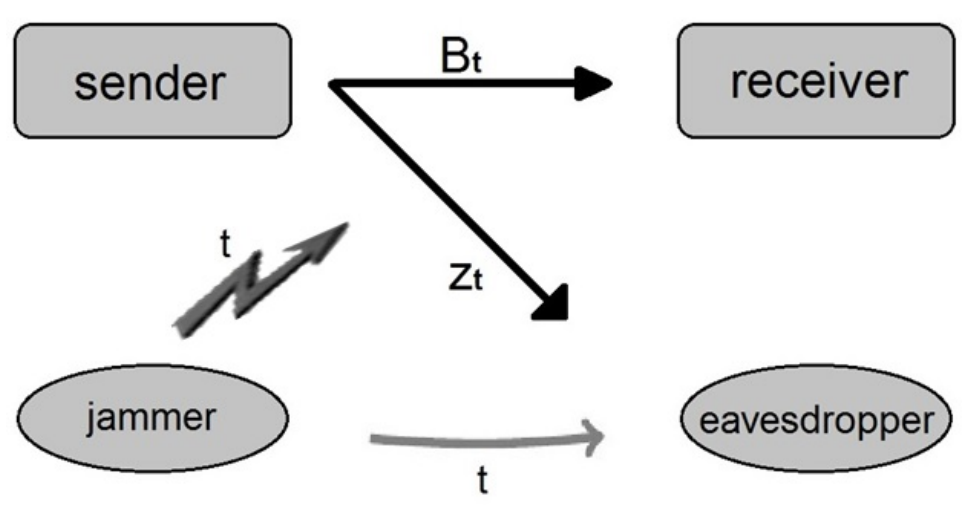

Figure 1: Arbitrarily varying classical-quantum wiretap channel

As aforementioned the lower bound in [11] and [12] is shown by building a two-part deterministic code. However that code concept still leaves something to be desired because we had to reduce the generality of the code concept when we explicitly allowed a small part of the code word to be non-secure. The code word we built was a composition of a public code word to synchronize the second part and a common randomness assisted code word to transmit the message. We only required security for the last part. As we will show in Corollary 4.1, when the jammer has access to the first part, it will be rendered completely useless. Thus the code concept only works when the jammer is limited in his action, e.g. we have to assume that eavesdropper cannot send messages towards the jammer. Nevertheless this code concept with weak criterion can be useful when small amount of public messages is desired, e.g. when the receiver uses it to estimate the channels. In this work we consider the general code concept when we construct a code in such a way that every part of it is secure. We show that when the legal channel is not symmetrizabel the sender can send a small amount of secure transmissions which push the secure capacity to the maximally attainable value. Thus the entire security is granted. We call it the strong code concept. This completes our analysis of arbitrarily varying classical-quantum wiretap channel.

In [11] we analyzed the secrecy capacities of various coding schemes with resource assistance. We showed that when the jammer was not allowed to has access to the resource, it was very helpful for the secure message transmission through an arbitrarily varying classical-quantum wiretap channel. In this work we analyze the case when the shared randomness is not secure against the jammer.

In [12] we showed that the secrecy capacity was in general not a continuous function of the system parameters. In [11] we proved super-activation for arbitrarily varying classical-quantum wiretap channels. In this work we establish 
complete characterizations for continuity and positivity of the capacity function of arbitrarily varying classical-quantum wiretap channels, and a complete characterization for super-activation.

This paper is organized as follows:

The main definitions are given in Section 2

In Section 3.1 we determine a secrecy capacity formula for a mixed channel model which is called the classic arbitrarily varying quantum wiretap channel. This formula is used for our result in Section 3.2

In Section 3.2 our main result is presented. In this section we determine the secrecy capacity for the arbitrarily varying classical-quantum channels under strong code concept.

In Section 4.1 we analyze when the sender and the legal receiver had the possibility to use shared randomness which is not secure against the jammer. We also determine the secrecy capacity of arbitrarily varying classical-quantum wiretap channels shared randomness which is secure against eavesdropping.

As an application of the results of our earlier works, in Section 4.2 we establish when the secrecy capacity of an arbitrarily varying classical-quantum wiretap channel is positive and when it is a continuous quantity of the system parameters. Furthermore we show when "super-activation" occurs for arbitrarily varying classical-quantum wiretap channels.

\section{Basic Definitions and Communication Scenar- ios}

For a finite set $\mathbf{A}$ we denote the set of probability distributions on $\mathbf{A}$ by $P(\mathbf{A})$. Let $\rho_{1}$ and $\rho_{2}$ be Hermitian operators on a finite-dimensional complex Hilbert space $G$. We say $\rho_{1} \geq \rho_{2}$ and $\rho_{2} \leq \rho_{1}$ if $\rho_{1}-\rho_{2}$ is positive-semidefinite. For a finite-dimensional complex Hilbert space $G$, we denote the (convex) space of density operators on $G$ by

$$
\mathcal{S}(G):=\left\{\rho \in \mathcal{L}(G): \rho \text { is Hermitian, } \rho \geq 0_{G}, \operatorname{tr}(\rho)=1\right\},
$$

where $\mathcal{L}(G)$ is the set of linear operators on $G$, and $0_{G}$ is the null matrix on $G$. Note that any operator in $\mathcal{S}(G)$ is bounded.

For finite sets $\mathbf{A}$ and $\mathbf{B}$, we define a (discrete) classical channel $\mathrm{V}: \mathbf{A} \rightarrow P(\mathbf{B})$, $\mathbf{A} \ni x \rightarrow \mathrm{V}(x) \in P(\mathbf{B})$ to be a system characterized by a probability transition matrix $\mathrm{V}(\cdot \cdot \cdot)$. For $x \in \mathbf{A}$ and $y \in \mathbf{B}, \mathbf{V}(y \mid x)$ expresses the probability of the output symbol $y$ when we send the symbol $x$ through the channel. The channel is said to be memoryless if the probability distribution of the output depends only on the input at that time and is conditionally independent of previous channel inputs and outputs. Further we can extend this definition when we define a classical channel to a map $\mathrm{V}: P(\mathbf{A}) \rightarrow P(\mathbf{B})$ by denoting $\mathrm{V}(y \mid p):=$ $\sum_{x \in \mathbf{A}} p(x) \mathrm{V}(y \mid x)$.

Let $n \in \mathbb{N}$. We define the $n$-th memoryless extension of the stochastic matrix $\mathrm{V}$ by $\mathrm{V}^{n}$, i.e., for $x^{n}=\left(x_{1}, \ldots, x_{n}\right) \in A^{n}$ and $y^{n}=\left(y_{1}, \ldots, y_{n}\right) \in B^{n}$, $\mathrm{V}^{n}\left(y^{n} \mid x^{n}\right)=\prod_{i=1}^{n} \mathrm{~V}\left(y_{i} \mid x_{i}\right)$.

For finite-dimensional complex Hilbert spaces $G$ and $G^{\prime}$ a quantum channel $N: \mathcal{S}(G) \rightarrow \mathcal{S}\left(G^{\prime}\right), \mathcal{S}(G) \ni \rho \rightarrow N(\rho) \in \mathcal{S}\left(G^{\prime}\right)$ is represented by a completely 
positive trace-preserving map which accepts input quantum states in $\mathcal{S}(G)$ and produces output quantum states in $\mathcal{S}\left(G^{\prime}\right)$.

If the sender wants to transmit a classical message of a finite set $A$ to the receiver using a quantum channel $N$, his encoding procedure will include a classical-to-quantum encoder to prepare a quantum message state $\rho \in \mathcal{S}(G)$ suitable as an input for the channel. If the sender's encoding is restricted to transmit an indexed finite set of quantum states $\left\{\rho_{x}: x \in \mathbf{A}\right\} \subset \mathcal{S}(G)$, then we can consider the choice of the signal quantum states $\rho_{x}$ as a component of the channel. Thus, we obtain a channel $\sigma_{x}:=N\left(\rho_{x}\right)$ with classical inputs $x \in \mathbf{A}$ and quantum outputs, which we call a classical-quantum channel. This is a map $\mathbf{N}: \mathbf{A} \rightarrow \mathcal{S}\left(G^{\prime}\right), \mathbf{A} \ni x \rightarrow \mathbf{N}(x) \in \mathcal{S}\left(G^{\prime}\right)$ which is represented by the set of $|\mathbf{A}|$ possible output quantum states $\left\{\sigma_{x}=\mathbf{N}(x):=N\left(\rho_{x}\right): x \in \mathbf{A}\right\} \subset \mathcal{S}\left(G^{\prime}\right)$, meaning that each classical input of $x \in \mathbf{A}$ leads to a distinct quantum output $\sigma_{x} \in \mathcal{S}\left(G^{\prime}\right)$. In view of this, we have the following definition.

Let $H$ be a finite-dimensional complex Hilbert space. A classical-quantum channel is a linear map $W: P(\mathbf{A}) \rightarrow \mathcal{S}(H), P(\mathbf{A}) \ni P \rightarrow W(P) \in \mathcal{S}(H)$. Let $a \in \mathbf{A}$. For a $P_{a} \in P(\mathbf{A})$, defined by $P_{a}\left(a^{\prime}\right)=\left\{\begin{array}{ll}1 & \text { if } a^{\prime}=a ; \\ 0 & \text { if } a^{\prime} \neq a\end{array}\right.$, , we write $W(a)$ instead of $W\left(P_{a}\right)$.

Remark 2.1. Frequently a classical-quantum channel is defined as a map $\mathbf{A} \rightarrow$ $\mathcal{S}(H), \mathbf{A} \ni a \rightarrow W(a) \in \mathcal{S}(H)$. This is a special case when the input is limited on the set $\left\{P_{a}: a \in \mathbf{A}\right\}$.

For any finite set $\mathbf{A}$, any finite-dimensional complex Hilbert space $H$, and $n \in \mathbb{N}$, we define $\mathbf{A}^{n}:=\left\{\left(a_{1}, \cdots, a_{n}\right): a_{i} \in \mathbf{A} \forall i \in\{1, \cdots, n\}\right\}$, and $H^{\otimes n}:=$ $\operatorname{span}\left\{v_{1} \otimes \cdots \otimes v_{n}: v_{i} \in H \forall i \in\{1, \cdots, n\}\right\}$. We also write $a^{n}$ for the elements of $\mathbf{A}^{n}$.

Let $n \in \mathbb{N}$. We define the $n$-th memoryless extension of the stochastic matrix $\mathrm{\vee}$ by $\mathrm{V}^{n}$, i.e., for $x^{n}=\left(x_{1}, \ldots, x_{n}\right) \in A^{n}$ and $y^{n}=\left(y_{1}, \ldots, y_{n}\right) \in B^{n}$, $\mathrm{V}^{n}\left(y^{n} \mid x^{n}\right)=\prod_{i=1}^{n} \mathrm{~V}\left(y_{i} \mid x_{i}\right)$.

We define the $n$-th extension of classical-quantum channel $W$ as follows. Associated with $W$ is the channel map on the n-block $W^{\otimes n}: P\left(\mathbf{A}^{n}\right) \rightarrow \mathcal{S}\left(H^{\otimes n}\right)$, such that $W^{\otimes n}\left(P^{n}\right)=W\left(P_{1}\right) \otimes \cdots \otimes W\left(P_{n}\right)$ if $P^{n} \in P\left(\mathbf{A}^{n}\right)$ can be written as $\left(P_{1}, \cdots, P_{n}\right)$.

Let $\theta:=\{1, \cdots, T\}$ be a finite set. Let $\left\{W_{t}: t \in \theta\right\}$ be a set of classicalquantum channels. For $t^{n}=\left(t_{1}, \cdots, t_{n}\right), t_{i} \in \theta$ we define the n-block $W_{t^{n}}$ such that for $W_{t^{n}}\left(P^{n}\right)=W_{t_{1}}\left(P_{1}\right) \otimes \cdots \otimes W_{t_{n}}\left(P_{n}\right)$ if $P^{n} \in P\left(\mathbf{A}^{n}\right)$ can be written as $\left(P_{1}, \cdots, P_{n}\right)$.

For a discrete random variable $X$ on a finite set $\mathbf{A}$ and a discrete random variable $Y$ on a finite set $\mathbf{B}$ we denote the Shannon entropy of $X$ by $H(X)=-\sum_{x \in \mathbf{A}} p(x) \log p(x)$ and the mutual information between $X$ and $Y$ by $I(X ; Y)=\sum_{x \in \mathbf{A}} \sum_{y \in \mathbf{B}} p(x, y) \log \left(\frac{p(x, y)}{p(x) p(y)}\right)$. Here $p(x, y)$ is the joint probability distribution function of $X$ and $Y$, and $p(x)$ and $p(y)$ are the marginal probability distribution functions of $X$ and $Y$ respectively, and "log" means logarithm to base 2 . 
For a quantum state $\rho \in \mathcal{S}(H)$ we denote the von Neumann entropy of $\rho$ by

$$
S(\rho)=-\operatorname{tr}(\rho \log \rho),
$$

where "log" means logarithm to base 2 .

Let $\mathfrak{P}$ and $\mathfrak{Q}$ be quantum systems. We denote the Hilbert space of $\mathfrak{P}$ and $\mathfrak{Q}$ by $G^{\mathfrak{P}}$ and $G^{\mathfrak{Q}}$, respectively. Let $\phi^{\mathfrak{P Q}}$ be a bipartite quantum state in $\mathcal{S}\left(G^{\mathfrak{P Q}}\right)$. We denote the partial trace over $G^{\mathfrak{P}}$ by

$$
\operatorname{tr}_{\mathfrak{P}}\left(\phi^{\mathfrak{P Q}}\right):=\sum_{l}\left\langle\left. l\right|_{\mathfrak{P}} \phi^{\mathfrak{P Q}} \mid l\right\rangle_{\mathfrak{P}}
$$

where $\left\{|l\rangle_{\mathfrak{P}}: l\right\}$ is an orthonormal basis of $G^{\mathfrak{P}}$. We denote the conditional entropy by

$$
S(\mathfrak{P} \mid \mathfrak{Q})_{\rho}:=S\left(\phi^{\mathfrak{P Q}}\right)-S\left(\phi^{\mathfrak{Q}}\right) .
$$

Here $\phi^{\mathfrak{Q}}=\operatorname{tr}_{\mathfrak{P}}\left(\phi^{\mathfrak{P Q}}\right)$. Let $\mathrm{V}: A \rightarrow \mathcal{S}(G)$ be a classical-quantum channel. For $P \in P(A)$ the conditional entropy of the channel for $\mathrm{V}$ with input distribution $P$ is denoted by

$$
S(\mathrm{v} \mid P):=\sum_{x \in A} P(x) S(\mathrm{v}(x)) .
$$

Let $\Phi:=\left\{\rho_{x}: x \in \mathbf{A}\right\}$ be a set of quantum states labeled by elements of $\mathbf{A}$. For a probability distribution $Q$ on $\mathbf{A}$, the Holevo $\chi$ quantity is defined as

$$
\chi(Q ; \Phi):=S\left(\sum_{x \in \mathbf{A}} Q(x) \rho_{x}\right)-\sum_{x \in \mathbf{A}} Q(x) S\left(\rho_{x}\right) .
$$

Note that we can always associate a state $\rho^{X Y}=\sum_{x} Q(x)|x\rangle\langle x| \otimes \rho_{x}$ to $(Q ; \Phi)$ such that $\chi(Q ; \Phi)=I(X ; Y)$ holds for the quantum mutual information. For a set $\mathbf{A}$ and a Hilbert space $G$ let $\mathbf{V}: \mathbf{A} \rightarrow \mathcal{S}(G)$ be a classical-quantum channel. For a probability distribution $P$ on A the Holevo $\chi$ quantity of the channel for $\mathbf{V}$ with input distribution $P$ is defined as

$$
\chi(P ; \Phi):=S(\mathbf{V}(P))-S(\mathbf{V} \mid P) .
$$

We denote the identity operator on a space $H$ by $\mathrm{id}_{H}$ and the symmetric group on $\{1, \cdots, n\}$ by $\mathrm{S}_{n}$.

For a probability distribution $P$ on a finite set $\mathbf{A}$ and a positive constant $\delta$, we denote the set of typical sequences by

$$
\mathcal{T}_{P, \delta}^{n}:=\left\{a^{n} \in \mathbf{A}^{n}:\left|\frac{1}{n} N\left(a^{\prime} \mid a^{n}\right)-P\left(a^{\prime}\right)\right| \leq \frac{\delta}{|\mathbf{A}|} \forall a^{\prime} \in \mathbf{A}\right\},
$$

where $N\left(a^{\prime} \mid a^{n}\right)$ is the number of occurrences of the symbol $a^{\prime}$ in the sequence $a^{n}$.

Let $G$ be a finite-dimensional complex Hilbert space. Let $n \in \mathbb{N}$ and $\alpha>0$. We suppose $\rho \in \mathcal{S}(G)$ has the spectral decomposition $\rho=\sum_{x} P(x)|x\rangle\langle x|$, its $\alpha$-typical subspace is the subspace spanned by $\left\{\left|x^{n}\right\rangle, x^{n} \in \mathcal{T}_{P, \alpha}^{n}\right\}$, where $\left|x^{n}\right\rangle:=$ $\otimes_{i=1}^{n}\left|x_{i}\right\rangle$. The orthogonal subspace projector onto the typical subspace is

$$
\Pi_{\rho, \alpha}=\sum_{x^{n} \in \mathcal{T}_{P, \alpha}^{n}}\left|x^{n}\right\rangle\left\langle x^{n}\right| .
$$


Similarly let $\mathbf{A}$ be a finite set, and $G$ be a finite-dimensional complex Hilbert space. Let V: $\mathbf{A} \rightarrow \mathcal{S}(G)$ be a classical-quantum channel. For $a \in \mathbf{A}$ suppose $\mathrm{V}(a)$ has the spectral decomposition $\mathrm{V}(a)=\sum_{j} V(j \mid a)|j\rangle\langle j|$ for a stochastic matrix $V(\cdot \mid \cdot)$. The $\alpha$-conditional typical subspace of $\mathrm{V}$ for a typical sequence $a^{n}$ is the subspace spanned by $\left\{\bigotimes_{a \in \mathbf{A}}\left|j^{\mathrm{I}_{a}}\right\rangle, j^{\mathrm{I}_{a}} \in \mathcal{T}_{V(\cdot \mid a), \delta}^{\mathrm{I}_{a}}\right\}$. Here $\mathrm{I}_{a}:=\{i \in$ $\left.\{1, \cdots, n\}: a_{i}=a\right\}$ is an indicator set that selects the indices $i$ in the sequence $a^{n}=\left(a_{1}, \cdots, a_{n}\right)$ for which the $i$-th symbol $a_{i}$ is equal to $a \in \mathbf{A}$. The subspace is often referred as the $\alpha$-conditional typical subspace of the state $\mathrm{V}^{\otimes n}\left(a^{n}\right)$. The orthogonal subspace projector onto it is defined as

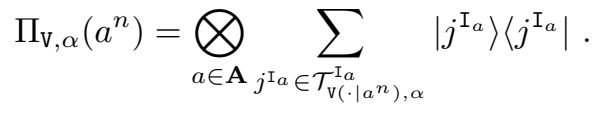

The typical subspace has following properties:

For $\sigma \in \mathcal{S}\left(G^{\otimes n}\right)$ and $\alpha>0$ there are positive constants $\beta(\alpha), \gamma(\alpha)$, and $\delta(\alpha)$, depending on $\alpha$ such that

$$
\begin{gathered}
\operatorname{tr}\left(\sigma \Pi_{\sigma, \alpha}\right)>1-2^{-n \beta(\alpha)}, \\
2^{n(S(\sigma)-\delta(\alpha))} \leq \operatorname{tr}\left(\Pi_{\sigma, \alpha}\right) \leq 2^{n(S(\sigma)+\delta(\alpha))}, \\
2^{-n(S(\sigma)+\gamma(\alpha))} \Pi_{\sigma, \alpha} \leq \Pi_{\sigma, \alpha} \sigma \Pi_{\sigma, \alpha} \leq 2^{-n(S(\sigma)-\gamma(\alpha))} \Pi_{\sigma, \alpha} .
\end{gathered}
$$

For $a^{n} \in \mathcal{T}_{P, \alpha}^{n}$ there are positive constants $\beta(\alpha)^{\prime}, \gamma(\alpha)^{\prime}$, and $\delta(\alpha)^{\prime}$, depending on $\alpha$ such that

$$
\begin{gathered}
\operatorname{tr}\left(\mathrm{V}^{\otimes n}\left(a^{n}\right) \Pi_{\mathrm{V}, \alpha}\left(a^{n}\right)\right)>1-2^{-n \beta(\alpha)^{\prime}} \\
2^{-n\left(S(\mathrm{~V} \mid P)+\gamma(\alpha)^{\prime}\right)} \Pi_{\mathrm{V}, \alpha}\left(a^{n}\right) \leq \Pi_{\mathrm{V}, \alpha}\left(a^{n}\right) \mathrm{V}^{\otimes n}\left(a^{n}\right) \Pi_{\mathrm{V}, \alpha}\left(a^{n}\right) \\
\leq 2^{-n\left(S(\mathrm{~V} \mid P)-\gamma(\alpha)^{\prime}\right)} \Pi_{\mathrm{V}, \alpha}\left(a^{n}\right) \\
2^{n\left(S(\mathrm{~V} \mid P)-\delta(\alpha)^{\prime}\right)} \leq \operatorname{tr}\left(\Pi_{\mathrm{V}, \alpha}\left(a^{n}\right)\right) \leq 2^{n\left(S(\mathrm{~V} \mid P)+\delta(\alpha)^{\prime}\right)} .
\end{gathered}
$$

For the classical-quantum channel V $: P(\mathbf{A}) \rightarrow \mathcal{S}(G)$ and a probability distribution $P$ on $\mathbf{A}$ we define a quantum state $P \mathrm{~V}:=\mathrm{V}(P)$ on $\mathcal{S}(G)$. For $\alpha>0$ we define an orthogonal subspace projector $\Pi_{P \mathrm{~V}, \alpha}$ fulfilling (1), (2), and (3). Let $x^{n} \in \mathcal{T}_{P, \alpha}^{n}$. For $\Pi_{P \mathrm{v}, \alpha}$ there is a positive constant $\beta(\alpha)^{\prime \prime}$ such that following inequality holds:

$$
\operatorname{tr}\left(\mathrm{V}^{\otimes n}\left(x^{n}\right) \cdot \Pi_{P \mathrm{~V}, \alpha}\right) \geq 1-2^{-n \beta(\alpha)^{\prime \prime}} .
$$

We give here a sketch of the proof. For a detailed proof please see [25].

Proof. (1) holds because $\operatorname{tr}\left(\sigma \Pi_{\sigma, \alpha}\right)=\operatorname{tr}\left(\Pi_{\sigma, \alpha} \sigma \Pi_{\sigma, \alpha}\right)=P\left(\mathcal{T}_{P, \alpha}^{n}\right)$. 2 holds because $\operatorname{tr}\left(\Pi_{\sigma, \alpha}\right)=\left|\mathcal{T}_{P, \alpha}^{n}\right| \cdot 3$ holds because $2^{-n(S(\sigma)+\gamma(\alpha))} \leq P^{n}\left(x^{n}\right) \leq 2^{-n(S(\sigma)-\gamma(\alpha))}$ for $x \in \mathcal{T}_{P, \alpha}^{n}$ and a positive $\gamma(\alpha)$. (4), (5), and (6) can be obtained in a similar way. (7) follows from the permutation-invariance of $\Pi_{P \mathrm{v}, \alpha}$. 
Definition 2.2. Let $\mathbf{A}$ and $\mathbf{B}$ be finite sets, and $H$ be a finite-dimensional complex Hilbert spaces. Let $\theta:=\{1, \ldots, T\}$ be a finite set. For every $t \in \theta$ let $\mathrm{W}_{t}$ be a classical channel $P(\mathbf{A}) \rightarrow P(\mathbf{B})$, and $W_{t}$ be a classical-quantum channel $P(\mathbf{A}) \rightarrow \mathcal{S}(H)$. We call the set of the classical channels $\left\{\mathrm{W}_{t}: t \in \theta\right\}$ an arbitrarily varying channel and the set of the classical-quantum channels $\left\{W_{t}: t \in \theta\right\}$ an arbitrarily varying classical-quantum channel when the channel state $t$ varies from symbol to symbol in an arbitrary manner.

Strictly speaking, the set $\left\{W_{t}: t \in \theta\right\}$ generates the arbitrarily varying classical-quantum channel $\left\{W_{t^{n}}: t^{n} \in \theta^{n}\right\}$. When the sender inputs a $P^{n} \in$ $P\left(\mathbf{A}^{n}\right)$ into the channel, the receiver receives the output $W_{t^{n}}\left(P^{n}\right) \in \mathcal{S}\left(H^{\otimes n}\right)$, where $t^{n}=\left(t_{1}, t_{2}, \cdots, t_{n}\right) \in \theta^{n}$ is the channel state of $W_{t^{n}}$.

Definition 2.3. We say that the arbitrarily varying channel $\left\{\mathrm{W}_{t}: t \in \theta\right\}$ is symmetrizable if there exists a parametrized set of distributions $\{\tau(\cdot \mid a): a \in \mathbf{A}\}$ on $\theta$ such that for all $a, a^{\prime} \in \mathbf{A}$, and $b \in \mathbf{B}$

$$
\sum_{t \in \theta} \tau(t \mid a) \mathrm{W}_{t}\left(b \mid a^{\prime}\right)=\sum_{t \in \theta} \tau\left(t \mid a^{\prime}\right) \mathrm{W}_{t}(b \mid a) .
$$

We say that the arbitrarily varying classical-quantum channel $\left\{W_{t}: t \in \theta\right\}$ is symmetrizable if there exists a parametrized set of distributions $\{\tau(\cdot \mid a): a \in \mathbf{A}\}$ on $\theta$ such that for all $a, a^{\prime} \in \mathbf{A}$,

$$
\sum_{t \in \theta} \tau(t \mid a) W_{t}\left(a^{\prime}\right)=\sum_{t \in \theta} \tau\left(t \mid a^{\prime}\right) W_{t}(a)
$$

Definition 2.4. Let $\mathbf{A}$ and $\mathbf{B}$ be finite sets, and $H$ be a finite-dimensional complex Hilbert spaces. Let $\theta:=\{1,2, \cdots\}$ be an index set. For every $t \in \theta$ let $\mathbf{W}_{t}$ be a classical channel $P(\mathbf{A}) \rightarrow P(\mathbf{B})$ and $V_{t}$ be a classical-quantum channel $P(\mathbf{A}) \rightarrow \mathcal{S}(H)$. We call the set of the classical/classical-quantum channel pairs $\left\{\left(\mathrm{W}_{t}, V_{t}\right): t \in \theta\right\}$ an classic arbitrarily varying quantum wiretap channel, when the state $t$ varies from symbol to symbol in an arbitrary manner, while the legitimate receiver accesses the output of the first channel, i.e., $W_{t}$ in the pair $\left(\mathrm{W}_{t}, V_{t}\right)$, and the wiretapper observes the output of the second channel, i.e., $V_{t}$ in the pair $\left(\mathrm{W}_{t}, V_{t}\right)$, respectively.

Definition 2.5. Let $\mathbf{A}$ be a finite set. Let $H$ and $H^{\prime}$ be finite-dimensional complex Hilbert spaces. Let $\theta:=\{1,2, \cdots\}$ be an index set. For every $t \in \theta$ let $W_{t}$ be a classical-quantum channel $P(\mathbf{A}) \rightarrow \mathcal{S}(H)$ and $V_{t}$ be a classical-quantum channel $P(\mathbf{A}) \rightarrow \mathcal{S}\left(H^{\prime}\right)$. We call the set of the classical-quantum channel pairs $\left\{\left(W_{t}, V_{t}\right): t \in \theta\right\}$ an arbitrarily varying classical-quantum wiretap channel, when the state $t$ varies from symbol to symbol in an arbitrary manner, while the legitimate receiver accesses the output of the first channel, i.e., $W_{t}$ in the pair $\left(W_{t}, V_{t}\right)$, and the wiretapper observes the output of the second channel, i.e., $V_{t}$ in the pair $\left(W_{t}, V_{t}\right)$, respectively.

Definition 2.6. An $\left(n, J_{n}\right)$ (deterministic) code $\mathcal{C}$ for the arbitrarily varying classical-quantum wiretap channel $\left\{\left(W_{t}, V_{t}\right): t \in \theta\right\}$ consists of a stochastic encoder $E:\left\{1, \cdots, J_{n}\right\} \rightarrow P\left(\mathbf{A}^{n}\right), j \rightarrow E(\cdot \mid j)$, specified by a matrix of conditional probabilities $E(\cdot \mid \cdot)$, and a collection of positive-semidefinite operators $\left\{D_{j}: j \in\left\{1, \cdots, J_{n}\right\}\right\}$ on $H^{\otimes n}$, which is a partition of the identity, i.e. $\sum_{j=1}^{J_{n}} D_{j}=\mathrm{id}_{H \otimes n}$. We call these operators the decoder operators. 
A code is created by the sender and the legal receiver before the message transmission starts. The sender uses the encoder to encode the message that he wants to send, while the legal receiver uses the decoder operators on the channel output to decode the message.

Remark 2.7. An $\left(n, J_{n}\right)$ deterministic code $\mathcal{C}$ with deterministic encoder consists of a family of $n$-length strings of symbols $\left(c_{j}\right)_{j \in\left\{1, \cdots, J_{n}\right\}} \in\left(\mathbf{A}^{n}\right)^{J_{n}}$ and a collection of positive-semidefinite operators $\left\{D_{j}: j \in\left\{1, \cdots, J_{n}\right\}\right\}$ on $H^{\otimes n}$ which is a partition of the identity.

The deterministic encoder is a special case of the stochastic encoder when we require that for every $j \in\left\{1, \cdots, J_{n}\right\}$, there is a sequence $a^{n} \in \mathbf{A}^{n}$ chosen with probability 1 . The standard technique for message transmission over a channel and robust message transmission over an arbitrarily varying channel is to use the deterministic encoder (cf. [3] and [13]). However, we use the stochastic encoder, since it is a tool for secure message transmission over wiretap channels (cf. [9] and [4]).

Definition 2.8. A non-negative number $R$ is an achievable secrecy rate for the arbitrarily varying classical-quantum wiretap channel $\left\{\left(W_{t}, V_{t}\right): t \in \theta\right\}$ if for every $\epsilon>0, \delta>0, \zeta>0$ and sufficiently large $n$ there exist an $\left(n, J_{n}\right)$ code $\mathcal{C}=\left(E,\left\{D_{j}^{n}: j=1, \cdots J_{n}\right\}\right)$ such that $\frac{\log J_{n}}{n}>R-\delta$, and

$$
\begin{gathered}
\max _{t^{n} \in \theta^{n}} P_{e}\left(\mathcal{C}, t^{n}\right)<\epsilon, \\
\max _{t^{n} \in \theta^{n}} \chi\left(R_{\text {uni }} ; Z_{t^{n}}\right)<\zeta,
\end{gathered}
$$

where $R_{\text {uni }}$ is the uniform distribution on $\left\{1, \cdots J_{n}\right\}$. Here $P_{e}\left(\mathcal{C}, t^{n}\right)$ (the average probability of the decoding error of a deterministic code $\mathcal{C}$, when the channel state of the arbitrarily varying classical-quantum wiretap channel $\left\{\left(W_{t}, V_{t}\right): t \in \theta\right\}$ is $\left.t^{n}=\left(t_{1}, t_{2}, \cdots, t_{n}\right)\right)$, is defined as

$$
P_{e}\left(\mathcal{C}, t^{n}\right):=1-\frac{1}{J_{n}} \sum_{j=1}^{J_{n}} \operatorname{tr}\left(W_{t^{n}}(E(\mid j)) D_{j}\right),
$$

$Z_{t^{n}}=\left\{V_{t^{n}}(E(\mid i)): i \in\left\{1, \cdots, J_{n}\right\}\right\}$ is the set of the resulting quantum state at the output of the wiretap channel when the channel state of $\left\{\left(W_{t}, V_{t}\right): t \in \theta\right\}$ is $t^{n}$.

The supremum on achievable secrecy rate for the $\left\{\left(W_{t}, V_{t}\right): t \in \theta\right\}$ is called the secrecy capacity of $\left\{\left(W_{t}, V_{t}\right): t \in \theta\right\}$ denoted by $C_{s}\left(\left\{\left(W_{t}, V_{t}\right): t \in \theta\right\}\right)$.

Remark 2.9. A weaker and widely used security criterion is obtained if we replace (9) with $\max _{t \in \theta} \frac{1}{n} \chi\left(R_{\text {uni }} ; Z_{t^{n}}\right)<\zeta$.

Remark 2.10. When we defined $W_{t}$ as $\mathbf{A} \rightarrow \mathcal{S}(H)$ then $P_{e}\left(\mathcal{C}, t^{n}\right)$ is defined as $1-\frac{1}{J_{n}} \sum_{j=1}^{J_{n}} \sum_{a^{n} \in \mathbf{A}^{n}} E\left(a^{n} \mid j\right) \operatorname{tr}\left(W_{t^{n}}\left(a^{n}\right) D_{j}\right)$.

When deterministic encoder is used then $P_{e}\left(\mathcal{C}, t^{n}\right)$ is defined as $1-\frac{1}{J_{n}} \sum_{j=1}^{J_{n}}$ $\operatorname{tr}\left(W_{t^{n}}\left(c_{j}\right) D_{j}\right)$. 
Definition 2.11. We denote the set of $\left(n, J_{n}\right)$ deterministic codes by $\Lambda$. A nonnegative number $R$ is an achievable secrecy rate for the arbitrarily varying classical-quantum wiretap channel $\left\{\left(W_{t}, V_{t}\right): t \in \theta\right\}$ under randomness assisted coding if for every $\delta>0, \zeta>0$, and $\epsilon>0$, if $n$ is sufficiently large, there is an $s$ a distribution $G$ on $(\Lambda, \sigma)$ such that $\frac{\log J_{n}}{n}>R-\delta$, and

$$
\begin{gathered}
\max _{t^{n} \in \theta^{n}} \int_{\Lambda} P_{e}\left(\mathcal{C}^{\gamma}, t^{n}\right) d G(\gamma)<\epsilon, \\
\max _{t^{n} \in \theta^{n}} \int_{\Lambda} \chi\left(R_{u n i}, Z_{\mathcal{C}^{\gamma}, t^{n}}\right) d G(\gamma)<\zeta .
\end{gathered}
$$

Here $\sigma$ is a sigma-algebra so chosen such that the functions $\gamma \rightarrow P_{e}\left(\mathcal{C}^{\gamma}, t^{n}\right)$ and $\gamma \rightarrow \chi\left(R_{\text {uni }} ; Z_{\mathcal{C}^{\gamma}, t^{n}}\right)$ are both $G$-measurable with respect to $\sigma$ for every $t^{n} \in \theta^{n}$

$A$ non-negative number $R$ is an achievable secrecy rate for the arbitrarily varying classical-quantum wiretap channel $\left\{\left(W_{t}, V_{t}\right): t \in \theta\right\}$ under nonsecure randomness assisted coding if for every $\delta>0, \zeta>0$, and $\epsilon>0$, if $n$ is sufficiently large, there is an $s$ a distribution $G$ on $(\Lambda, \sigma)$ such that $\frac{\log J_{n}}{n}>R-\delta$, and

$$
\begin{gathered}
\int_{\Lambda} \max _{t^{n} \in \theta^{n}} P_{e}\left(\mathcal{C}^{\gamma}, t^{n}\right) d G(\gamma)<\epsilon, \\
\int_{\Lambda} \max _{t^{n} \in \theta^{n}} \chi\left(R_{u n i}, Z_{\mathcal{C}^{\gamma}, t^{n}}\right) d G(\gamma)<\zeta .
\end{gathered}
$$

Here $\sigma$ is a sigma-algebra so chosen such that the functions $\gamma \rightarrow \max _{t^{n} \in \theta^{n}} P_{e}\left(\mathcal{C}^{\gamma}, t^{n}\right)$ and $\gamma \rightarrow \max _{t^{n} \in \theta^{n}} \chi\left(R_{u n i} ; Z_{\mathcal{C}^{\gamma}, t^{n}}\right)$ are both $G$-measurable with respect to $\sigma$.

The supremum on achievable secrecy rate for the $\left\{\left(\mathrm{W}_{t}, V_{t}\right): t \in \theta\right\}$ under randomness assisted coding is called the randomness assisted secrecy capacity of $\left\{\left(\mathrm{W}_{t}, V_{t}\right): t \in \theta\right\}$ denoted by $C_{s}\left(\left\{\left(\mathrm{~W}_{t}, V_{t}\right): t \in \theta\right\}, r\right)$. The supremum on achievable secrecy rate for the $\left\{\left(\mathrm{W}_{t}, V_{t}\right): t \in \theta\right\}$ under non-secure randomness assisted coding is called the non-secure randomness assisted secrecy capacity of $\left\{\left(\mathrm{W}_{t}, V_{t}\right): t \in \theta\right\}$ denoted by $C_{s}\left(\left\{\left(\mathrm{~W}_{t}, V_{t}\right): t \in \theta\right\}, r_{n s}\right)$.

Definition 2.12. An $\left(n, J_{n}\right)$ code $\mathcal{C}$ for the classic arbitrarily varying quantum wiretap channel $\left\{\left(\mathrm{W}_{t}, V_{t}\right): t \in \theta\right\}$ consists of a stochastic encoder $E$ : $\left\{1, \cdots, J_{n}\right\} \rightarrow P\left(\mathbf{A}^{n}\right), j \rightarrow E(\cdot \mid j)$, specified by a matrix of conditional probabilities $E(\cdot \mid \cdot)$, and a collection of mutually disjoint sets $\left\{D_{j} \subset \mathbf{B}^{n}: j \in\left\{1, \ldots, J_{n}\right\}\right\}$ (decoding sets).

Definition 2.13. A non-negative number $R$ is an achievable secrecy rate for the classic arbitrarily varying quantum wiretap channel $\left\{\left(\mathrm{W}_{t}, V_{t}\right): t \in \theta\right\}$ if for every $\epsilon>0, \delta>0, \zeta>0$ and sufficiently large $n$ there exist an $\left(n, J_{n}\right)$ code $\mathcal{C}=\left(E,\left\{D_{j}: j=1, \cdots J_{n}\right\}\right)$ such that $\frac{\log J_{n}}{n}>R-\delta$, and

$$
\frac{1}{J_{n}} \max _{t \in \theta} \sum_{j=1}^{J_{n}} \mathrm{~W}_{t}^{n}\left(D_{j}^{c} \mid E(\cdot \mid j)\right) \leq \varepsilon,
$$

and

$$
\max _{t^{n} \in \theta^{n}} \chi\left(R_{u n i} ; Z_{t^{n}}\right)<\zeta .
$$

The supremum on achievable secrecy rate for the $\left\{\left(\mathrm{W}_{t}, V_{t}\right): t \in \theta\right\}$ is called the secrecy capacity of $\left\{\left(\mathrm{W}_{t}, V_{t}\right): t \in \theta\right\}$ denoted by $C_{s}\left(\left\{\left(\mathrm{~W}_{t}, V_{t}\right): t \in \theta\right\}\right)$. 
Definition 2.14. A non-negative number $R$ is an achievable secrecy rate for the arbitrarily varying classical-quantum wiretap channel $\left\{\left(W_{t}, V_{t}\right): t \in \theta\right\}$ under common randomness assisted quantum coding using an amount $g_{n}$ of secret common randomness, where $g_{n}$ is a non-negative number depending on $n$, if for every $\delta>0, \zeta>0, \epsilon>0$, and sufficiently large $n$, there is a set of $\left(n, J_{n}\right)$ codes $\left(\left\{\mathcal{C}^{\gamma}: \gamma \in \Gamma_{n}\right\}\right)$ such that $\frac{1}{n} \log \left|\Gamma_{n}\right|=g_{n}, \frac{\log J_{n}}{n}>R-\delta$, and

$$
\begin{gathered}
\max _{t^{n} \in \theta^{n}} \frac{1}{2^{n g_{n}}} \sum_{\gamma=1}^{2^{n g_{n}}} P_{e}\left(\mathcal{C}^{\gamma}, t^{n}\right)<\epsilon, \\
\max _{t^{n} \in \theta^{n}} \chi\left(R_{\text {uni }}, Z_{t^{n}}\right)<\zeta,
\end{gathered}
$$

where $R_{\text {uni }}$ is the uniform distribution on $\left\{1, \cdots J_{n}\right\}$.

Unlike in [11] and [12] we require that the randomness to be secure against eavesdropping here.

The supremum on achievable secrecy rate under random assisted quantum coding using an amount $g_{n}$ of common randomness of $\left\{\left(W_{t}, V_{t}\right): t \in \theta\right\}$ is called the secret random assisted secrecy capacity of $\left\{\left(W_{t}, V_{t}\right): t \in \theta\right\}$ using an amount $g_{n}$ of common randomness, denoted by $C_{k e y}\left(\left\{\left(W_{t}, V_{t}\right): t \in \theta\right\} ; g_{n}\right)$.

Definition 2.15. We say super-activation occurs to two arbitrarily varying classicalquantum wiretap channels $\left\{\left(W_{t}, V_{t}\right): t \in \theta\right\}$ and $\left\{\left(W^{\prime}{ }_{t}, V^{\prime}{ }_{t}\right): t \in \theta\right\}$ when the following hold:

$$
\begin{gathered}
C_{s}\left(\left\{\left(W_{t}, V_{t}\right): t \in \theta\right\}\right)=0, \\
C_{s}\left(\left\{\left(W^{\prime}{ }_{t}, V^{\prime}{ }_{t}\right): t \in \theta\right\}\right)=0,
\end{gathered}
$$

and

$$
C_{s}\left(\left\{W_{t} \otimes W^{\prime}{ }_{t^{\prime}}, V_{t} \otimes V_{t^{\prime}}^{\prime}: t, t^{\prime} \in \theta\right\}\right)>0 .
$$

Remark 2.16. For super-activation we do not require the strong code concept.

\section{Strong Code Concept}

\subsection{Classic Arbitrarily Varying Quantum Wiretap Chan- nel}

At first we determine a capacity formula for a mixed channel model, i.e. the secrecy capacity of classic arbitrarily varying quantum wiretap channel. This formula will be used for our result for secrecy capacity of arbitrarily varying classical-quantum wiretap channels using secretly sent common randomness.

Theorem 3.1. Let $\left\{\left(\grave{W}_{t}, V_{t}\right): t \in \theta\right\}$ be a classic arbitrarily varying quantum wiretap channel. When $\left\{\grave{W}_{t}: t \in \theta\right\}$ is not symmetrizable, then

$$
C_{s}\left(\left\{\left(\grave{W}_{t}, V_{t}\right): t \in \theta\right\}\right)=\lim _{n \rightarrow \infty} \frac{1}{n} \max _{U \rightarrow A \rightarrow\left\{B_{q}^{n}, Z_{t}: q, t_{n}\right.} \min _{q \in P(\theta)} I\left(p_{U}, \grave{B}_{q}^{n}\right)-\max _{t^{n} \in \theta^{n}} \chi\left(p_{U}, Z_{t^{n}}\right) .
$$

Here $\grave{B}_{t}$ are the resulting classical random variables at the output of the legitimate receiver's channels and $Z_{t^{n}}$ are the resulting quantum states at the output of wiretap channels. The maximum is taken over all random variables 
that satisfy the Markov chain relationships: $U \rightarrow A \rightarrow \grave{B}_{q} Z_{t}$ for every $\grave{B}_{q} \in$ $\operatorname{Conv}\left(\left(\grave{B}_{t}\right)_{t \in \theta}\right)$ and $t \in \theta$. A is here a random variable taking values on $\mathbf{A}, U$ a random variable taking values on some finite set $\mathbf{U}$ with probability distribution $p_{U}$.

Proof. We fix a probability distribution $p \in P(\mathbf{A})$ and choose an arbitrarily positive $\delta$. Let

$$
J_{n}=\left\lfloor 2^{\inf _{\grave{B}_{q} \in \operatorname{Conv}\left(\left(\grave{B}_{s}\right)_{s \in \theta}\right)} I\left(p ; \grave{B}_{q}^{n}\right)-\max _{t^{n} \in \theta^{n}} \chi\left(p ; Z_{t^{n}}\right)-n \delta}\right\rfloor,
$$

and

$$
L_{n}=\left\lceil 2^{\max _{t^{n} \in \theta^{n}}\left(\chi\left(p ; Z_{t^{n}}\right)+n \delta\right)} .\right\rceil
$$

Let $p^{\prime}\left(x^{n}\right):=\left\{\begin{array}{ll}\frac{p^{n}\left(x^{n}\right)}{p^{n}\left(\mathcal{T}_{p, \delta}^{n}\right)} & \text { if } x^{n} \in \mathcal{T}_{p, \delta}^{n} \\ 0 & \text { else . }\end{array} ;\right.$

and $X^{n}:=\left\{X_{j, l}\right\}_{j \in\left\{1, \cdots, J_{n}\right\}, l \in\left\{1, \cdots, L_{n}\right\}}$ be a family of random matrices whose components are i.i.d. according to $p^{\prime}$.

We fix a $t^{n} \in \theta^{n}$ and define a map $\vee: P(\theta) \times P(\mathbf{A}) \rightarrow \mathcal{S}(H)$ by

$$
\mathrm{V}(t, p):=V_{t}(p)
$$

For $t \in \theta$ we define $q(t):=\frac{N\left(t \mid t^{n}\right)}{n}$. $t^{n}$ is trivially a typical sequence of $q$. For $p \in P(\mathbf{A}), \mathrm{V}$ defines a map $\mathrm{V}(\cdot, p): P(\theta) \rightarrow \mathcal{S}(H)$.

Let

$$
Q_{t^{n}}\left(x^{n}\right):=\Pi_{\mathrm{V}(\cdot, p), \alpha}\left(t^{n}\right) \Pi_{\mathrm{V}, \alpha}\left(t^{n}, x^{n}\right) \cdot V_{t^{n}}\left(x^{n}\right) \cdot \Pi_{\mathrm{V}, \alpha}\left(t^{n}, x^{n}\right) \Pi_{\mathrm{V}(\cdot, p), \alpha}\left(t^{n}\right) .
$$

Lemma 3.2 (Gentle Operator, cf. [26] and [21]). Let $\rho$ be a quantum state and $X$ be a positive operator with $X \leq \mathrm{I}$ and $1-\operatorname{tr}(\rho X) \leq \lambda \leq 1$. Then

$$
\|\rho-\sqrt{X} \rho \sqrt{X}\|_{1} \leq \sqrt{2 \lambda}
$$

The Gentle Operator was first introduced in 26], where it has been shown that $\|\rho-\sqrt{X} \rho \sqrt{X}\|_{1} \leq \sqrt{8 \lambda}$. In [21], the result of [26] has been improved, and (12) has been proved.

In view of the fact that $\Pi_{\mathrm{V}(\cdot, p), \alpha}\left(t^{n}\right)$ and $\Pi_{\mathrm{V}, \alpha}\left(t^{n}, x^{n}\right)$ are both projection matrices, by (1), (7), and Lemma 3.2 for any $t$ and $x^{n}$, it holds that

$$
\left\|Q_{t^{n}}\left(x^{n}\right)-V_{t^{n}}\left(x^{n}\right)\right\|_{1} \leq \sqrt{2^{-n \beta(\alpha)}+2^{-n \beta(\alpha)^{\prime \prime}}} .
$$

The following Lemma has been showed in [12]:

Lemma 3.3 (Alternative Covering Lemma). Let $\mathcal{V}$ be a finite-dimensional Hilbert space. Let $\mathrm{M}$ and $\mathrm{M}^{\prime} \subset \mathrm{M}$ be finite sets. Suppose we have an ensemble $\left\{\rho_{m}: m \in \mathrm{M}\right\} \subset \mathcal{S}(\mathcal{V})$ of quantum states. Let $p$ be a probability distribution on M.

Suppose a total subspace projector $\Pi$ and codeword subspace projectors $\left\{\Pi_{m}\right.$ : $m \in \mathrm{M}\}$ exist which project onto subspaces of the Hilbert space in which the states 
exist, and for all $m \in \mathrm{M}^{\prime}$ there are positive constants $\left.\epsilon \in\right] 0,1[, D, d$ such that the following conditions hold:

$$
\begin{gathered}
\operatorname{tr}\left(\rho_{m} \Pi\right) \geq 1-\epsilon, \\
\operatorname{tr}\left(\rho_{m} \Pi_{m}\right) \geq 1-\epsilon, \\
\operatorname{tr}(\Pi) \leq D,
\end{gathered}
$$

and

$$
\Pi_{m} \rho_{m} \Pi_{m} \leq \frac{1}{d} \Pi_{m} .
$$

We denote $\omega:=\sum_{m \in \mathrm{M}^{\prime}} p(m) \rho_{m}$. Notice that $\omega$ is not a density operator in general. We define a sequence of i.i.d. random variables $X_{1}, \ldots, X_{L}$, taking values in $\left\{\rho_{m}: m \in \mathrm{M}\right\}$. If $L \gg \frac{d}{D}$ then

$$
\begin{aligned}
& \operatorname{Pr}\left(\left\|L^{-1} \sum_{i=1}^{L} \Pi \cdot \Pi_{X_{i}} \cdot X_{i} \cdot \Pi_{X_{i}} \cdot \Pi-\omega\right\|_{1}\right. \\
& \left.\leq 1-p\left(\mathrm{M}^{\prime}\right)+4 \sqrt{1-p\left(\mathrm{M}^{\prime}\right)}+42 \sqrt[8]{\epsilon}\right) \\
& \geq 1-2 D \exp \left(-p\left(\mathrm{M}^{\prime}\right) \frac{\epsilon^{3} L d}{2 \ln 2 D}\right) .
\end{aligned}
$$

By (2) we have

$$
\begin{aligned}
& \operatorname{tr}\left(\Pi_{\mathrm{V}(\cdot, p), \alpha}\left(t^{n}\right)\right) \\
& \leq 2^{n(S(\mathrm{~V}(\cdot, p) \mid q)+\delta(\alpha))} \\
& =2^{n\left(\sum_{t} q(t) \mathrm{V}(t, p)+\delta(\alpha)\right)} \\
& =2^{n\left(\sum_{t} q(t) S\left(V_{t}(p)\right)+\delta(\alpha)\right) .} .
\end{aligned}
$$

Furthermore, for all $x^{n}$ it holds that

$$
\begin{aligned}
& \Pi_{\mathrm{V}, \alpha}\left(t^{n}, x^{n}\right) V_{t^{n}}\left(x^{n}\right) \Pi_{\mathrm{V}, \alpha}\left(t^{n}, x^{n}\right) \\
& \leq 2^{-n\left(S(\mathrm{~V} \mid r)+\delta(\alpha)^{\prime}\right)} \Pi_{\mathrm{V}, \alpha}\left(t^{n}, x^{n}\right) \\
& =2^{-n\left(\sum_{t, x} r(t, x) S(\mathrm{~V}(t, x))+\delta(\alpha)^{\prime}\right)} \Pi_{\mathrm{V}, \alpha}\left(t^{n}, x^{n}\right) .
\end{aligned}
$$

We define

$$
\theta^{\prime}:=\{t \in \theta: n q(t) \geq \sqrt{n}\}
$$

By properties of classical typical set (cf. [26] ) there is a positive $\hat{\beta}(\alpha)$ such that

$$
\underset{p^{\prime}}{\operatorname{Pr}}\left(x^{n} \in\left\{x^{n} \in \mathbf{A}^{n}:\left(x_{\mathrm{I}_{t}}\right) \in \mathrm{T}_{p, \delta}^{n q(t)} \forall t \in \theta^{\prime}\right\}\right) \geq\left(1-2^{-\sqrt{n} \hat{\beta}(\alpha)}\right)^{|\theta|} \geq 1-2^{-\sqrt{n} \frac{1}{2} \hat{\beta}(\alpha)},
$$

where $\mathrm{I}_{t}:=\left\{i \in\{1, \cdots, n\}: t_{i}=t\right\}$ is an indicator set that selects the indices $i$ in the sequence $t^{n}=\left(t_{1}, \cdots, t_{n}\right)$. 
We denote the set $\left\{x^{n}:\left(x_{\mathrm{I}_{t}}\right) \in \mathrm{T}_{p, \delta}^{n q(t)} \forall t \in \theta^{\prime}\right\} \subset \mathbf{A}^{n}$ by $\mathbf{M}_{t^{n}}$. For all $x^{n} \in \mathrm{M}_{t^{n}}$, if $n$ is sufficiently large, we have

$$
\begin{aligned}
& \left|\sum_{t, x} r(t, x) S(\mathrm{~V}(t, x))-\sum_{t} q(t) S\left(V_{t} \mid p\right)\right| \\
& \leq\left|\sum_{t \in \theta^{\prime}, x} r(t, x) S(\mathrm{~V}(t, x))-\sum_{t \in \theta^{\prime}} q(t) S\left(V_{t} \mid p\right)\right| \\
& +\left|\sum_{t \notin \theta^{\prime}, x} r(t, x) S(\mathrm{~V}(t, x))-\sum_{t \notin \theta^{\prime}} q(t) S\left(V_{t} \mid p\right)\right| \\
& \leq \sum_{t \in \theta^{\prime}}\left|\sum_{x} r(t, x) S(\mathrm{~V}(t, x))-q(t) S\left(V_{t} \mid p\right)\right|+2|\theta| \frac{1}{\sqrt{n}} C \\
& \leq 2|\theta| \frac{\delta}{n} C+2|\theta| \frac{1}{\sqrt{n}} C,
\end{aligned}
$$

where $C:=\max _{t \in \theta} \max _{x \in \mathbf{A}}\left(S(\mathrm{~V}(t, x))+S\left(V_{t} \mid p\right)\right)$. We set $\Theta_{t^{n}}:=\sum_{x^{n} \in \mathrm{M}_{t} n} p\left(x^{n}\right) Q_{t^{n}}\left(x^{n}\right)$. For given $z^{n} \in \mathrm{M}_{t^{n}}$ and $t^{n} \in \theta^{n},\left\langle z^{n}\left|\Theta_{t^{n}}\right| z^{n}\right\rangle$ is the expected value of $\left\langle z^{n}\left|Q_{t^{n}}\left(x^{n}\right)\right| z^{n}\right\rangle$ under the condition $x^{n} \in \mathrm{M}_{t^{n}}$.

We choose a positive $\bar{\beta}(\alpha)$ such that $\bar{\beta}(\alpha) \leq \min \left(2^{-n \beta(\alpha)}, 2^{-n \beta(\alpha)^{\prime}}\right)$, and set $\epsilon:=2^{-n \bar{\beta}(\alpha)}$. In view of 16 we now apply Lemma 3.3 , where we consider the set $\mathrm{M}_{t^{n}} \subset \mathbf{A}^{n}$ : If $n$ is sufficiently large, for all $j$ we have

$$
\begin{aligned}
& \operatorname{Pr}\left(\left\|\sum_{l=1}^{L_{n}} \frac{1}{L_{n}} Q_{t^{n}}\left(X_{j, l}\right)-\Theta_{t^{n}}\right\|_{1}>2^{-\sqrt{n} \frac{1}{8} \hat{\beta}(\alpha)}+40 \sqrt[8]{\epsilon}\right) \\
& \leq 2^{n\left(\sum_{t, x} r(t, x) S(\mathrm{~V}(t, x))+\delta(\alpha)\right)} \\
& \cdot \exp \left(-L_{n} \frac{\epsilon^{3}}{2 \ln 2}\left(1-2^{-\sqrt{n} \frac{1}{2} \hat{\beta}(\alpha)}\right) \cdot 2^{n\left(\sum_{t} q(t) S\left(V_{t}(p)\right)-\sum_{t} q(t) S\left(V_{t} \mid p\right)\right)+\delta(\alpha)+\delta(\alpha)^{\prime}+2|\theta| \frac{\delta}{n} C+2|\theta| \frac{1}{\sqrt{n}} C}\right) \\
& =2^{n\left(\sum_{t, x} r(t, x) S(\mathrm{~V}(t, x))+\delta(\alpha)\right.} \\
& \cdot \exp \left(-L_{n} \frac{\epsilon^{3}}{2 \ln 2} \cdot\left(1-2^{-\sqrt{n} \frac{1}{2} \hat{\beta}(\alpha)}\right) 2^{n\left(-\sum_{t} q(t) \chi\left(p ; Z_{t}\right)+\delta(\alpha)+\delta(\alpha)^{\prime}+2|\theta| \frac{\delta}{n} C+2|\theta| \frac{1}{\sqrt{n}} C\right)}\right) .
\end{aligned}
$$

The equality holds since $S\left(V_{t}(p)\right)-S\left(V_{t} \mid p\right)=\chi\left(p ; Z_{t}\right)$.

$$
\text { Furthermore, }
$$

$$
\begin{aligned}
& \operatorname{Pr}\left(\left\|\sum_{l=1}^{L_{n}} \frac{1}{L_{n}} Q_{t^{n}}\left(X_{j, l}\right)-\Theta_{t^{n}}\right\|_{1}>2^{-\sqrt{n} \frac{1}{8} \hat{\beta}(\alpha)}+40 \sqrt[8]{\epsilon} \forall t^{n} \forall j\right) \\
& \leq J_{n}|\theta|^{n} 2^{n\left(\sum_{t, x} r(t, x) S(\mathrm{~V}(t, x))+\delta(\alpha)\right.} \\
& \cdot \exp \left(-L_{n} \frac{\epsilon^{3}}{2 \ln 2}\left(1-2^{-\sqrt{n} \frac{1}{2} \hat{\beta}(\alpha)}\right) 2^{n\left(-\sum_{t} q(t) \chi\left(p ; Z_{t}\right)+\delta(\alpha)+\delta(\alpha)^{\prime}+2|\theta| \frac{\delta}{n} C+2|\theta| \frac{1}{\sqrt{n}} C\right)}\right) .
\end{aligned}
$$


Let $\phi_{t}^{j}$ be the quantum state at the output of wiretapper's channel when the channel state is $t$ and $j$ has been sent. We have

$$
\begin{aligned}
& \sum_{t \in \theta} q(t) \chi\left(p ; Z_{t}\right)-\chi\left(p ; \sum_{t} q(t) Z_{t}\right) \\
& =\sum_{t \in \theta} q(t) S\left(\sum_{j=1}^{J_{n}} \frac{1}{J_{n}} \phi_{t}^{j}\right)-\sum_{t \in \theta} \sum_{j=1}^{J_{n}} q(t) \frac{1}{J_{n}} S\left(\phi_{t}^{j}\right) \\
& -S\left(\frac{1}{J_{n}} \sum_{t \in \theta} \sum_{j=1}^{J_{n}} q(t) \phi_{t}^{j}\right)+\sum_{j=1}^{J_{n}} \frac{1}{J_{n}} S\left(\sum_{t \in \theta} q(t) \phi_{t}^{j}\right) .
\end{aligned}
$$

Let $H^{\mathfrak{T}}$ be a $|\theta|$-dimensional Hilbert space spanned by an orthonormal basis $\{|t\rangle: t=1, \cdots,|\theta|\}$. Let $H^{\mathfrak{J}}$ be a $J_{n}$-dimensional Hilbert space spanned by an orthonormal basis $\left\{|j\rangle: j=1, \cdots, J_{n}\right\}$. We define

$$
\varphi^{\mathfrak{J} \mathfrak{T} H^{n}}:=\frac{1}{J_{n}} \sum_{j=1}^{J_{n}} \sum_{t \in \theta} q(t)|j\rangle\langle j|\otimes| t\rangle\langle t| \otimes \phi_{t}^{j} .
$$

We have

$$
\begin{gathered}
\varphi^{\mathfrak{J} H^{n}}=\operatorname{tr}_{\mathfrak{T}}\left(\varphi^{\mathfrak{J} \mathfrak{T} H^{n}}\right)=\frac{1}{J_{n}} \sum_{j=1}^{J_{n}} \sum_{t \in \theta} q(t)|j\rangle\langle j| \otimes \phi_{t}^{j} ; \\
\varphi^{\mathfrak{T} H^{n}}=\operatorname{tr}_{\mathfrak{J}}\left(\varphi^{\mathfrak{J} \mathfrak{T} H^{n}}\right)=\frac{1}{J_{n}} \sum_{j=1}^{J_{n}} \sum_{t \in \theta} q(t)|t\rangle\langle t| \otimes \phi_{t}^{j} ; \\
\varphi^{H^{n}}=\operatorname{tr}_{\mathfrak{J}} \mathfrak{T}\left(\varphi^{\mathfrak{J} \mathfrak{T} H^{n}}\right)=\frac{1}{J_{n}} \sum_{j=1}^{J_{n}} \sum_{t \in \theta} q(t) \phi_{t}^{j} .
\end{gathered}
$$

Thus,

$$
\begin{gathered}
S\left(\varphi^{\mathfrak{J} H^{n}}\right)=H\left(R_{\text {uni }}\right)+\frac{1}{J_{n}} \sum_{j=1}^{J_{n}} S\left(\sum_{t \in \theta} q(t) \phi_{t}^{j}\right) ; \\
S\left(\varphi^{\mathfrak{T} H^{n}}\right)=H\left(Y_{q}\right)+\sum_{t \in \theta} q(t) S\left(\frac{1}{J_{n}} \sum_{j=1}^{J_{n}} \phi_{t}^{j}\right) ; \\
S\left(\varphi^{\mathfrak{J} \mathfrak{T} H^{n}}\right)=H\left(R_{\text {uni }}\right)+H\left(Y_{q}\right)+\frac{1}{J_{n}} \sum_{j=1}^{J_{n}} \sum_{t \in \theta} q(t) S\left(\phi_{t}^{j}\right),
\end{gathered}
$$

where $Y_{q}$ is a random variable on $\theta$ with distribution $q(t)$.

By strong subadditivity of von Neumann entropy, it holds that $S\left(\varphi^{\mathfrak{J} H^{n}}\right)+$ $S\left(\varphi^{\mathfrak{T} H^{n}}\right) \geq S\left(\varphi^{H^{n}}\right)+S\left(\varphi^{\mathfrak{j} H^{n}}\right)$, therefore

$$
\sum_{t} q(t) \chi\left(p ; Z_{t}\right)-\chi\left(p ; \sum_{t} q(t) Z_{t}\right) \geq 0 .
$$


For an arbitrary $\zeta$, we define $L_{n}=\left\lceil 2^{\max _{t^{n}} \chi\left(p ; Z_{t^{n}}\right)+n \zeta}\right\rceil$, and choose a suitable $\alpha, \bar{\beta}(\alpha)$, and sufficiently large $n$ such that $6 \bar{\beta}(\alpha)+2 \delta(\alpha)+2 \delta(\alpha)^{\prime}+2|\theta| \frac{\delta}{n} C$ $+2|\theta| \frac{1}{\sqrt{n}} C \leq \zeta$. By $\left[21\right.$, if $n$ is sufficiently large, we have $L_{n} \geq\left\lceil 2^{n\left(\sum_{t} q(t) \chi\left(p ; Z_{t}\right)+\zeta\right)}\right\rceil$ and

$$
L_{n} \frac{\epsilon^{3}}{2 \ln 2}\left(1-2^{-\sqrt{n} \frac{1}{2} \hat{\beta}(\alpha)}\right) 2^{n\left(-\sum_{t} q(t) \chi\left(p ; Z_{t}\right)+\delta(\alpha)+\delta(\alpha)^{\prime}+2|\theta| \frac{\delta}{n} C+2|\theta| \frac{1}{\sqrt{n}} C\right)}>2^{\frac{1}{2} n \zeta} .
$$

When $n$ is sufficiently large for any positive $\vartheta$ it holds that

$$
\begin{aligned}
& J_{n}|\theta|^{n} 2^{n\left(\sum_{t, x} r(t, x) S(\mathrm{~V}(t, x))+\delta(\alpha)\right.} \exp \left(-2^{\frac{1}{4} n \zeta}\right) \\
& \leq 2^{-n \vartheta}
\end{aligned}
$$

and

$$
2^{-\sqrt{n} \frac{1}{8} \hat{\beta}(\alpha)}+40 \sqrt[8]{\epsilon} \leq 2^{-\sqrt{n} \frac{1}{16} \hat{\beta}(\alpha)} .
$$

Thus for sufficiently large $n$ we have

$$
\begin{aligned}
& \operatorname{Pr}\left(\left\|\sum_{l=1}^{L_{n}} \frac{1}{L_{n}} Q_{t^{n}}\left(X_{j, l}\right)-\Theta_{t^{n}}\right\|_{1} \leq 2^{-\sqrt{n} \frac{1}{16} \hat{\beta}(\alpha)} \forall t^{n} \forall j\right) \\
& \geq 1-2^{n \vartheta}
\end{aligned}
$$

for any positive $\varrho$.

In [15], the following was shown: Let $\left\{X_{j, l}\right\}_{j \in\left\{1, \ldots, J_{n}\right\}, l \in\left\{1, \ldots, L_{n}\right\}}$ be a family of random variables taking value according to $p^{\prime}$. We assume $\left\{\grave{W}_{t}: t \in \theta\right\}$ is not symmetrizable. If $n$ is sufficiently large, and if $J_{n} \cdot L_{n} \leq 2^{\left.i n f_{\grave{B}_{q} \in \operatorname{Conv}\left(\left(\grave{B}_{t}\right)_{t \in \theta}\right)} I\left(p ; \grave{B}_{q}\right)-\mu\right)}$ for an arbitrary positive $\mu$ there exists a set of mutually disjoint $\operatorname{sets}\left\{D_{j, l}: j \in\right.$ $\left\{1, \cdots, J_{n}\right\}, l \in\left\{1, \cdots, L_{n}\right\}$ on $\mathbf{B}^{n}$ such that for all positive $\epsilon, t^{n} \in \theta^{n}$, and $j \in\left\{1, \ldots, J_{n}\right\}$

$$
\underset{p^{\prime}}{\operatorname{Pr}}\left[\operatorname{tr}\left(\grave{W}_{t^{n}}\left(X_{j, l}\right) D_{j, l}\right) \geq 1-2^{-n \beta}\right]>1-2^{-n \gamma}
$$

By 22 and 23, when $\left\{\grave{W}_{t}: t \in \theta\right\}$ is not symmetrizable we can find with positive probability a realization $x_{j, l}$ of $X_{j, l}$ and set of mutually disjoint sets $\left\{D_{j, l}: j \in\left\{1, \cdots, J_{n}\right\}, l \in\left\{1, \cdots, L_{n}\right\}\right.$ such that for all positive $\epsilon, t^{n} \in \theta^{n}$, and $j \in\left\{1, \ldots, J_{n}\right\}$

$$
\max _{t \in \theta} \frac{1}{J_{n}} \sum_{j=1}^{J_{n}} \grave{W}_{t^{n}}\left(D_{j, l}^{c} \mid x_{j, l}\right) \leq \epsilon,
$$

and

$$
\left\|\sum_{l=1}^{L_{n}} \frac{1}{L_{n}} Q_{t^{n}}\left(x_{j, l}\right)-\Theta_{t^{n}}\right\|_{1} \leq \epsilon .
$$

Here we define $E\left(x^{n} \mid j\right)=\frac{1}{L_{n}}$ if $x^{n} \in\left\{x_{j, l}: l \in\left\{1, \ldots, L_{n}\right\}\right.$,

We choose a suitable positive $\alpha$. For any given $j^{\prime} \in\left\{1, \ldots, J_{n}\right\}$, by 13 and 
(25) we have

$$
\begin{aligned}
& \left\|\sum_{l=1}^{L_{n}} \frac{1}{L_{n}} V_{t^{n}}\left(x_{j^{\prime}, l}\right)-\Theta_{t^{n}}\right\|_{1} \\
& \leq\left\|\sum_{l=1}^{L_{n}} \frac{1}{L_{n}} V_{t^{n}}\left(x_{j^{\prime}, l}\right)-\sum_{l=1}^{L_{n}} \frac{1}{L_{n}} Q_{t^{n}}\left(x_{j^{\prime}, l}\right)\right\|_{1} \\
& \quad+\left\|\sum_{l=1}^{L_{n}} \frac{1}{L_{n}} Q_{t^{n}}\left(x_{j^{\prime}, l}\right)-\Theta_{t^{n}}\right\|_{1} \\
& \leq 2^{-\sqrt{n} \frac{1}{16} \hat{\beta}(\alpha)}+\sqrt{2^{-\frac{1}{2} n \beta(\alpha)}+2^{-\frac{1}{2} n \beta(\alpha)^{\prime \prime}}} \\
& \leq 2^{-\sqrt{n} \frac{1}{32} \hat{\beta}(\alpha)} .
\end{aligned}
$$

Notice that by 26 we have $\left\|\frac{1}{J_{n} \cdot L_{n}} \sum_{j=1}^{J_{n}} \sum_{l=1}^{L_{n}} V_{t^{n}}\left(x_{j, l}\right)-\Theta_{t^{n}}\right\|_{1} \leq 2^{-\sqrt{n} \frac{1}{32} \hat{\beta}(\alpha)}$.

Lemma 3.4 (Fannes-Audenaert Ineq., cf. [18], [5]). Let $\Phi$ and $\Psi$ be two quantum states in a d-dimensional complex Hilbert space and $\|\Phi-\Psi\| \leq \mu<\frac{1}{e}$, then

$$
|S(\Phi)-S(\Psi)| \leq \mu \log (d-1)+h(\mu),
$$

where $h(\nu):=-\nu \log \nu-(1-\nu) \log (1-\nu)$ for $\nu \in[0,1]$.

The Fannes Inequality was first introduced in 18, where it has been shown that $|S(\mathfrak{X})-S(\mathfrak{Y})| \leq \mu \log d-\mu \log \mu$. In [5] the result of [18] has been improved, and (27) has been proved.

By Lemma 3.4 and the inequality (26), for a uniformly distributed random variable $R_{\text {uni }}$ with values in $\left\{1, \ldots, J_{n}\right\}$ a and $t^{n} \in \theta^{n}$, we have

$$
\begin{aligned}
& \chi\left(R_{\text {uni }} ; Z_{t^{n}}\right) \\
& =S\left(\sum_{j=1}^{J_{n}} \frac{1}{J_{n}} \sum_{l=1}^{L_{n}} \frac{1}{L_{n}} V_{t^{n}}\left(x_{j, l}\right)\right) \\
& -\sum_{j=1}^{J_{n}} \frac{1}{J_{n}} S\left(\sum_{l=1}^{L_{n}} \frac{1}{L_{n}} V_{t^{n}}\left(\pi\left(x_{j, l}\right)\right)\right) \\
& \leq\left|S\left(\sum_{j=1}^{J_{n}} \frac{1}{J_{n}} \sum_{l=1}^{L_{n}} \frac{1}{L_{n}} V_{t^{n}}\left(x_{j, l}\right)\right)-S\left(\Theta_{t^{n}}\right)\right| \\
& +\left|S\left(\Theta_{t^{n}}\right)-\sum_{j=1}^{J_{n}} \frac{1}{J_{n}} S\left(\sum_{l=1}^{L_{n}} \frac{1}{L_{n}} V_{t^{n}}\left(x_{j, l}\right)\right)\right| \\
& \leq 2 \cdot 2^{-\sqrt{n} \frac{1}{32} \hat{\beta}(\alpha)} \log (n d-1)+2 h\left(2^{-\sqrt{n} \frac{1}{32} \hat{\beta}(\alpha)}\right) .
\end{aligned}
$$

By 28, for any positive $\lambda$ if $n$ is sufficiently large, we have

$$
\chi\left(R_{u n i} ; Z_{t^{n}}\right) \leq \lambda
$$


We define $E\left(x^{n} \mid j\right)=\left\{\begin{array}{l}\frac{1}{L_{n}} \text { if } x^{n} \in\left\{x_{j, l}: l \in\left\{1, \ldots, L_{n}\right\}\right\} \\ 0 \text { if } x \notin\left\{x_{j, l}: l \in\left\{1, \ldots, L_{n}\right\}\right\} .\end{array} \quad\right.$ and $D_{j}:=$ $\bigcup_{l} D_{j, l}$. By 25$)$ and 29 , when $\left\{\grave{W}_{t}: t \in \theta\right\}$ is not symmetrizable the deterministic secrecy capacity of $\left\{\left(\grave{W}_{t}, V_{t}\right): t \in \theta\right\}$ is larger or equal to

$$
\lim _{n \rightarrow \infty} \frac{1}{n}\left(\inf _{B_{q} \in \operatorname{Conv}\left(\left(B_{s}\right)_{s \in \theta}\right)} \chi\left(p ; \grave{B}_{q}^{n}\right)-\max _{t^{n} \in \theta^{n}} \chi\left(p ; Z_{t^{n}}\right)\right)-\varepsilon .
$$

The achievability of $\lim _{n \rightarrow \infty} \frac{1}{n} \max _{U \rightarrow A \rightarrow\left\{B_{q}, Z_{t}: q, t\right\}}\left(\inf _{\grave{B}_{q} \in \operatorname{Conv}\left(\left(\grave{B}_{s}\right)_{s \in \theta}\right)} I\left(p_{U} ; \grave{B}_{q}\right)\right.$ $\left.-\max _{t^{n} \in \theta^{n}} \chi\left(p_{U} ; Z_{t^{n}}\right)\right)$ and the converse are shown by the standard arguments (cf. [16] and [6]).

\subsection{The Secure Message Transmission With Strong Code Concept}

Now we are going to prove our main result: the secrecy capacity formula for arbitrarily varying classical-quantum wiretap channels using secretly sent common randomness. In our previous papers [11] and [12] we determined the secrecy capacity formula for arbitrarily varying classical-quantum wiretap channels. Our strategy is to build a two-part code word, which consists of a non-secure code word and a common randomness-assisted secure code word. The non-secure one is used to create the common randomness for the sender and the legal receiver. The common randomness-assisted secure code word is used to transmit the message to the legal receiver.

Now we build a code in such a way that the transmission of both the message and the randomization is secure. Since the technique introduced in [15] for classical channels cannot be easily transferred into quantum channels, our idea is to construct a classical arbitrarily varying quantum wiretap channel and apply Theorem 3.1. In [4 a technique has been introduced to construct a classical arbitrarily varying channel by means of an arbitrarily varying classical-quantum channel. However this technique does not work for classical arbitrarily varying quantum wiretap channel since it cannot provide security. We have to find a more sophisticated way.

Theorem 3.5. If the arbitrarily varying classical-quantum channel $\left\{W_{t}: t \in \theta\right\}$ is not symmetrizable, then

$C_{s}\left(\left\{\left(W_{t}, V_{t}\right): t \in \theta\right\}\right)=\lim _{n \rightarrow \infty} \frac{1}{n} \max _{U \rightarrow A \rightarrow\left\{B_{q}, Z_{t}: q, t\right\}}\left(\inf _{B_{q} \in \operatorname{Conv}\left(\left(B_{t}\right)_{t \in \theta}\right)} \chi\left(p_{U} ; B_{q}^{\otimes n}\right)-\max _{t^{n} \in \theta^{n}} \chi\left(p_{U} ; Z_{t^{n}}\right)\right)$,

when we use a two-part code word that both parts are secure.

Here $B_{t}$ are the resulting quantum states at the output of the legitimate receiver's channels. $Z_{t^{n}}$ are the resulting quantum states at the output of wiretap channels. The maximum is taken over all random variables that satisfy the Markov chain relationships: $U \rightarrow A \rightarrow B_{q} Z_{t}$ for every $B_{q} \in \operatorname{Conv}\left(\left(B_{t}\right)_{t \in \theta}\right)$ and $t \in \theta$. $A$ is here a random variable taking values on $\mathbf{A}, U$ a random variable taking values on some finite set $\mathbf{U}$ with probability distribution $p_{U}$.

Proof. Since the security of both the message and the randomization implies the security of only the message, the secrecy capacity of $\left\{\left(W_{t}, V_{t}\right): t \in \theta\right\}$ 
for the message and the randomization transmission cannot exceed $\lim _{n \rightarrow \infty} \frac{1}{n}$

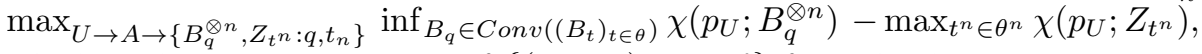
which is the secrecy capacity of $\left\{\left(W_{t}, V_{t}\right): t \in \theta\right\}$ for only the message transmission (cf. [12]). Thus the converse is trivial.

For the achievability we at first assume that $\left\{W_{t}: t \in \theta\right\}$ is symmetrizable. In this case the secrecy capacity of $\left\{\left(W_{t}, V_{t}\right): t \in \theta\right\}$ for only the message transmission is zero and there is nothing to prove. At next we assume that for all $p \in$ $P(\mathbf{U})$ we have $\lim _{n \rightarrow \infty} \frac{1}{n} \max _{U \rightarrow A \rightarrow\left\{B_{q}^{\otimes n}, Z_{t} n: q, t_{n}\right\}}\left(\inf _{B_{q} \in \operatorname{Conv}\left(\left(B_{t}\right)_{t \in \theta}\right)} \chi\left(p_{U} ; B_{q}^{\otimes n}\right)\right.$ $\left.-\max _{t^{n} \in \theta^{n}} \chi\left(p_{U} ; Z_{t^{n}}\right)\right) \leq 0$. In this case the secrecy capacity of $\left\{\left(W_{t}, V_{t}\right): t \in\right.$ $\theta\}$ for only the message transmission is also zero and again there is nothing to prove. Now we assume that $\left\{W_{t}: t \in \theta\right\}$ is not symmetrizable and for all sufficiently large $n$ and a positive $\epsilon$

$$
\frac{1}{n} \max _{U \rightarrow A \rightarrow\left\{B_{q}^{\otimes n}, Z_{\left.t^{n}: q, t_{n}\right\}}\right.}\left(\inf _{B_{q} \in \operatorname{Conv}\left(\left(B_{t}\right)_{t \in \theta}\right)} \chi\left(p_{U} ; B_{q}^{\otimes n}\right)-\max _{t^{n} \in \theta^{n}} \chi\left(p ; Z_{t^{n}}\right)\right)>2 \epsilon
$$

holds.

\section{i) Construction of a Non-Symmetrizable Channel with Random Pre-coding}

We consider the Markov chain $U \rightarrow A \rightarrow\left\{B_{q}, Z_{t}: q, t\right\}$, where we define the classical channel $P(\mathbf{U}) \rightarrow P(\mathbf{A})$ by $T_{U}$. It may happen that $\left\{W_{t} \circ T_{U}: t \in \theta\right\}$ is symmetrizable although $\left\{W_{t}: t \in \theta\right\}$ is not symmetrizable, as following example shows:

We assume that $\left\{W_{t}: t \in \theta\right\}: P(\mathbf{A}) \rightarrow \mathcal{S}(H)$ is not symmetrizable but there is a subset $\mathbf{A}^{\prime} \subset \mathbf{A}$ such that $\left\{W_{t}: t \in \theta\right\}$ limited on $A^{\prime}$ is symmetrizable. We choose a $T_{U}$ such that for every $u \in \mathbf{U}$ there is $a \in \mathbf{A}^{\prime}$ such that $T_{U}(a \mid u)=1$, and $T_{U}(a \mid u)=0$ for all $a \in \mathbf{A} \backslash \mathbf{A}^{\prime}$ and $u \in \mathbf{U}$. It is clear that $\left\{W_{t} \circ T_{U}: t \in \theta\right\}$ is symmetrizable (cf. also [20] for an example for classical channels).

We now use a technique introduced in [20] to overcome this: Without loss of generality we may assume that $|\mathbf{A}|=|\mathbf{U}|$ by optimization. Furthermore without loss of generality we may assume that $\mathbf{A}=\mathbf{U}$ by relabeling the symbols. For every $n>1 \in \mathbb{N}$ we define a new classical channel $\tilde{T}_{U}^{n}: P\left(\mathbf{A}^{n}\right) \rightarrow P\left(\mathbf{A}^{n}\right)$ by setting $\tilde{T}_{U}^{n}:=T_{U}^{n-1} \times i d_{\mathbf{A}}$, i.e.,

$$
\tilde{T}_{U}^{n}\left(a_{1}, \cdots, a_{n-1}, a_{n}\right):=T_{U}^{n}\left(a_{1}, \cdots, a_{n-1}\right) \cdot \delta_{a_{n}} .
$$

We have

$$
W_{t^{n}} \circ \tilde{T}_{U}^{n}\left(a_{1}, \cdots, a_{n-1}, a_{n}\right)=W_{t^{n-1}} \circ T_{U}^{n}\left(a_{1}, \cdots, a_{n-1}\right) W_{t_{n}}\left(a_{n}\right),
$$

where for $t^{n}=\left(t_{1}, \cdots, t_{n-1}, t_{n}\right)$ we denote $t^{n-1}:=\left(t_{1}, \cdots, t_{n-1}\right)$. Since $\left\{W_{t}\right.$ : $t \in \theta\}$ is not symmetrizable, $\left\{W_{t^{n}} \circ \tilde{T}_{U}^{n}: t \in \theta\right\}$ is not symmetrizable. Furthermore, for any positive $\delta$ sufficiently large $n$ we have

$C\left(\left\{W_{t^{n}} \circ T_{U}^{n}: t \in \theta\right\} ; r\right) \leq C\left(\left\{W_{t^{n-1}} \circ T_{U}^{n-1}: t \in \theta\right\} ; r\right)+\delta \leq C\left(\left\{W_{t^{n}} \circ \tilde{T}_{U}^{n-1}: t \in \theta\right\} ; r\right)+\delta$.

For every $n>1$ and $t^{n} \in \theta^{n}$ we define $\check{W}_{t^{n}}: P\left(\mathbf{U}^{n}\right) \rightarrow P\left(\mathbf{A}^{n}\right)$ by

$$
\check{W}_{t^{n}}:=W_{t^{n}} \circ \tilde{T}_{U}^{n} .
$$


This shows that, if for a non-symmetrizable channel $\left\{W_{t}: t \in \Theta\right\}$ we have (32), then

$$
\frac{1}{n} \max _{U \rightarrow A \rightarrow\left\{\check{B}_{q}^{\otimes n}, Z_{t^{n}}: q, t_{n}\right\}}\left(\inf _{\check{B}_{q} \in \operatorname{Conv}\left(\left(\check{B}_{t}\right)_{t \in \theta)}\right.} \chi\left(p_{U} ; \check{B}_{q}^{\otimes n}\right)-\max _{t^{n} \in \theta^{n}} \chi\left(p ; Z_{t^{n}}\right)\right)>\epsilon
$$

holds, where $\check{B}_{t^{n}}$ are the resulting quantum states at the output of $\check{W}_{t^{n}}$.

ii) Definition of a Classical Arbitrarily Varying Channel Which Is Not Symmetrizable

We denote $m:=\log n$ and define $\check{V}_{t}:=V_{t} \circ T_{U}$ for all $t \in \theta$. Now we consider the arbitrarily varying wiretap classical-quantum channel $\left\{\left(\check{W}_{t}, \check{V}_{t}\right) ; t \in \theta\right\}$. We choose an arbitrary $\delta>0$, by (34) if $m$ is sufficiently large we may assume that for at least one $p \in P(\mathbf{U})$

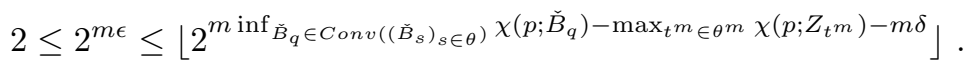

By Theorem 1 of [12] if $m$ is sufficiently large we can find a $(m, 2)$ code $\left(E^{m},\left\{D_{j}^{m}: j \in\{1,2\}\right\}\right)$ and positive $\lambda, \zeta$ such that for some $q \in P(\theta)$

$$
1-\frac{1}{2} \sum_{j=1}^{2} \operatorname{tr}\left(\check{W}_{q}^{\otimes m}\left(E^{m}(\mid j)\right) D_{j}^{m}\right) \geq 1-2^{-m^{1 / 16} \lambda}
$$

and for all $t^{m} \in \theta^{m}$ and $\pi \in \Pi_{m}$

$$
\left\|\check{V}_{t^{m}}\left(\pi\left(E^{m}(\mid j)\right)\right)-\Theta_{t^{m}}\right\|<2^{-\sqrt{m} \zeta}
$$

for a $\Theta_{t^{m}} \in \mathcal{S}\left(H^{m}\right)$ which is independent of $j$. Here for $\pi \in \mathrm{S}_{m}$ we define its permutation matrix on $H^{\otimes m}$ by $P_{\pi}$.

(Notice that $\left(E^{m},\left\{D_{j}^{m}: j \in\{1,2\}\right\}\right)$ is a deterministic code for a mixed channel model called compound-arbitrarily varying wiretap classical-quantum channel which we introduced in [12].)

We now combine a technique introduced in [4] with the concept of superposition code to define a set of classical channels.

We choose $d^{m^{2}}+1$ Hermitian operators $L_{i} \geq 0, i=1, \cdots, d^{m^{2}}+1$ which span the space of Hermitian operators on $H^{m}$ and fulfill $\sum_{i=1}^{d^{m}+1} L_{i}=i d_{H^{m}}$ by the technique introduced in [4]: We choose arbitrarily $d^{m 2}$ Hermitian operators $\bar{L}_{i} \geq 0, i=1, \cdots, d^{m 2}$ which span the space of Hermitian operators on $H^{m}$ and denote the trace of $\sum_{i=1}^{d^{m 2}} \bar{L}_{i}$ by $\lambda$. Now we define $L_{i}:=\frac{1}{\lambda} \bar{L}_{i}$ for $i \in\left\{1, \cdots, d^{m 2}\right\}$ and $L_{d^{m 2}+1}:=i d_{H^{m}}-\sum_{i=1}^{d^{m 2}} L_{i}$.

Now we defined the classical arbitrarily varying channel $\left\{\grave{W}_{t^{m}}: t^{m} \in \theta^{m}\right\}$ $: P\left(\mathbf{U}^{m}\right) \rightarrow P\left(\left\{1, \cdots, d^{m 2}+3\right\}\right)$ by

$$
\grave{W}_{t^{m}}\left(i \mid p^{m}\right):= \begin{cases}\frac{1}{2} \operatorname{tr}\left(\check{W}_{t^{m}}\left(p^{m}\right) D_{i}^{m}\right) & \text { for } i \in\{1,2\} \\ \frac{1}{2} \operatorname{tr}\left(\check{W}_{t^{m}}\left(p^{m}\right) L_{i-2}\right) & \text { for } i=3, \cdots, d^{m 2}+3 .\end{cases}
$$

Since $\frac{1}{2} \sum_{j=1}^{2} D_{j}^{m}+\frac{1}{2} \sum_{i=1}^{d^{m 2}+1} L_{i}=i d_{H^{m}}$ we have

$$
\sum_{i=1}^{d^{m 2}+3} \grave{W}_{t^{m}}\left(i \mid p^{m}\right)=1
$$


for all $p^{m} \in P\left(\mathbf{U}^{m}\right)$. Thus this definition is valid.

When $\left\{\grave{W}_{t^{m}}: t^{m} \in \theta^{m}\right\}$ is symmetrizable then there is a $\left\{\tau\left(\cdot \mid a^{m}\right): a^{m} \in\right.$ $\left.\mathbf{U}^{m}\right\}$ on $\theta^{m}$ such that

$$
\sum_{t^{m} \in \theta^{m}} \tau\left(t^{m} \mid a^{m}\right) \grave{W}_{t^{m}}\left(a^{\prime m}\right)=\sum_{t^{m} \in \theta^{m}} \tau\left(t^{m} \mid a^{\prime m}\right) \grave{W}_{t^{m}}\left(a^{m}\right)
$$

for all $i \in\left\{1, \cdots, d^{m 2}+3\right\}$. This implies that

$$
\frac{1}{2} \sum_{t^{m} \in \theta^{m}} \tau\left(t^{m} \mid a^{m}\right) \operatorname{tr}\left(\check{W}_{t^{m}}\left(a^{\prime m}\right) L_{i}\right)=\frac{1}{2} \sum_{t^{m} \in \theta^{m}} \tau\left(t^{m} \mid a^{\prime m}\right) \operatorname{tr}\left(\check{W}_{t^{m}}\left(a^{m}\right) L_{i}\right)
$$

for all $i \in\left\{1, \cdots, d^{m^{2}}+1\right\}$.

Since $\left\{L_{i}: i=1, \cdots, d^{m 2}\right\}$ span the space of Hermitian operators on $H^{m}$ we have

$$
\sum_{t^{m} \in \theta^{m}} \tau\left(t^{m} \mid a^{m}\right) \check{W}_{t^{m}}\left(a^{\prime m}\right)=\sum_{t^{m} \in \theta^{m}} \tau\left(t^{m} \mid a^{\prime m}\right) \check{W}_{t^{m}}\left(a^{m}\right) .
$$

This is a contradiction to our assumption that $\left\{\breve{W}_{t^{m}}: t^{m} \in \theta^{m}\right\}$ is not symmetrizable, therefore $\left\{\grave{W}_{t^{m}}: t^{m} \in \theta^{m}\right\}$ is not symmetrizable. tive

iii) The Deterministic Secrecy Capacity of $\left\{\left(\grave{W}_{t^{m}}, \check{V}_{t^{m}}\right): t^{m} \in \theta^{m}\right\}$ Is Posi-

By 35 for all $q \in P(\theta)$ and $j \in\{1,2\}$ we have

$$
\grave{W}_{q}(j \mid j) \geq \frac{1}{2}-\frac{1}{2} 2^{-m^{1 / 16} \lambda},
$$

and for all $q \in P(\theta)$ and $j \neq i \in\{1,2\}$

$$
\grave{W}_{q}(j \mid i) \leq \frac{1}{2} 2^{-m^{1 / 16} \lambda} .
$$

We denote the uniform distribution on $\{1,2\}$ by $R^{\prime}$. For any positive $\zeta^{\prime}$ if $m$ is sufficiently large by (37) and (38) for all $q \in P(\theta)$ we have

$$
\begin{aligned}
& \min _{q \in P\left(\theta^{m}\right)} I\left(E^{m}\left(\cdot \mid R^{\prime}\right), \grave{B}_{q}\right) \\
& >\left(\frac{1}{2}-\frac{1}{2} 2^{-m^{1 / 16} \lambda}\right) \log \left(\frac{1}{2}-\frac{1}{2} 2^{-m^{1 / 16} \lambda}\right)-\left(\frac{1}{2}+\frac{1}{2} 2^{-m^{1 / 16} \lambda}\right) \log \left(\frac{1}{4}+\frac{1}{4} 2^{-m^{1 / 16} \lambda}\right)-\zeta^{\prime} \\
& \geq \frac{1}{2}-2 \zeta^{\prime}
\end{aligned}
$$

where $\grave{B}_{q}$ is the resulting distribution at the output of $\grave{W}_{q}$.

Applying the Lemma 3.4 and (36) if $m$ is sufficiently large for any $n^{\prime} \in \mathbb{N}$, positive $\zeta^{\prime}$, and for all $t^{m n^{\prime}}=\left(t_{1}^{m}, \cdots, t_{n^{\prime}}^{m}\right)=\left(t_{1}, \cdots, t_{m}, t_{m+1}, \cdots, t_{2 m}, t_{2 m+1}, \cdots, t_{m n^{\prime}}\right)$ 


$$
\begin{aligned}
& \in \theta^{m n^{\prime}} \text { we have } \\
& \frac{1}{n^{\prime}} \max _{t^{m n^{\prime}} \in \theta^{m n^{\prime}}} \chi\left(R^{\otimes n^{\prime}}, \check{Z}_{t^{m n^{\prime}}}\right) \\
& =\frac{1}{n^{\prime}}\left(S\left(\frac{1}{2^{n^{\prime}}} \sum_{j \in\{1,2\}^{n^{\prime}}} \check{V}_{t^{m n^{\prime}}}\left(\left(E^{m}\right)^{\otimes n^{\prime}}(\cdot \mid j)\right)\right)-\frac{1}{2^{n^{\prime}}} \sum_{j \in\{1,2\}^{n^{\prime}}} S\left(\check{V}_{t^{m n^{\prime}}}\left(\left(E^{m}\right)^{\otimes n^{\prime}}(\cdot \mid j)\right)\right)\right) \\
& \leq \frac{1}{n^{\prime}}\left|S\left(\frac{1}{2^{n^{\prime}}} \sum_{j \in\{1,2\}^{n^{\prime}}} \check{V}_{t^{m n^{\prime}}}\left(\left(E^{m}\right)^{\otimes n^{\prime}}(\cdot \mid j)\right)\right)-S\left(\Theta_{t^{m n^{\prime}}}\right)\right| \\
& +\frac{1}{n^{\prime}}\left|S\left(\Theta_{t^{m n^{\prime}}}\right)-\frac{1}{2^{n^{\prime}}} \sum_{j \in\{1,2\}^{n^{\prime}}} S\left(\check{V}_{t^{m n^{\prime}}}\left(\left(E^{m}\right)^{\otimes n^{\prime}}(\cdot \mid j)\right)\right)\right| \\
& +\frac{1}{n^{\prime}} \mid \sum_{i=1}^{n^{\prime}}\left(S\left(\frac{1}{2} \sum_{j \in\{1,2\}} \check{V}_{t_{i}^{m}}\left(\left(E^{m}\right)(\cdot \mid j)\right)-S\left(\Theta_{t_{i}^{m}}\right)\right) \mid\right. \\
& \leq 2 \cdot \sum_{n^{\prime}}^{n^{\prime}}\left(S\left(\Theta_{t_{i}^{m}}\right)-\frac{1}{2} \sum_{j \in\{1,2\}} S\left(\check{V}_{t_{i}^{m}}\left(\left(E^{m}\right)(\cdot \mid j)\right)\right)\right) \mid \\
& \leq \zeta^{\prime},
\end{aligned}
$$

where $\check{Z}_{t^{m n^{\prime}}}$ is the resulting quantum state at the output of $\check{V}_{t m n^{\prime}}$.

We choose $\zeta^{\prime}<\frac{1}{18}$ and a sufficiently large $m$ such that $(39)$ and 40 hold. Sine $\left\{\grave{W}_{t^{m}}: t^{m} \in \theta^{m}\right\}$ is not symmetrizable, by Theorem 3.1 the deterministic secrecy capacity of $\left\{\left(\grave{W}_{t^{m}}, \check{V}_{t^{m}}\right): t^{m} \in \theta^{m}\right\}$ is equal to

$$
\limsup _{n^{\prime} \rightarrow \infty} \frac{1}{n^{\prime}} \max _{p \in P\left(\mathbf{U}^{m}\right)} \min _{q \in P\left(\theta^{m}\right)} I\left(p, \grave{B}_{q}^{n^{\prime}}\right)-\max _{t^{m n^{\prime}} \in \theta^{m n^{\prime}}} \chi\left(p, \check{Z}_{t^{m n^{\prime}}}\right) \geq \frac{1}{2}-3 \zeta^{\prime}>\frac{1}{3} \text {. }
$$

iv) The Secure Transmission of the Message with a Deterministic Code

Since $(\log n)^{2} \gg 3 \log \left(n^{3}\right)$ we can build a $\left((\log n)^{2}, n^{3}\right) \operatorname{code}\left(\tilde{E}^{(\log n)^{3}},\left\{\tilde{S}_{i}^{(\log n)^{3}}\right.\right.$ : $\left.\left.i \in\left\{1, \cdots, n^{3}\right\}\right\}\right)$ such that

$$
\begin{aligned}
& \quad 1-\min _{t^{(\log n)^{3}} \in \theta^{(\log n)^{3}}} \min _{i \in\left\{1, \cdots, n^{3}\right\}} \grave{W}_{t^{(\log n)^{3}}}\left(\tilde{S}_{i}^{(\log n)^{3}} \mid \tilde{E}^{(\log n)^{3}}(\cdot \mid i)\right) \\
& \leq \varepsilon
\end{aligned}
$$

and

$$
\max _{t^{(\log n)^{3} \in \theta^{(\log n)^{3}}}}\left\|\check{V}_{t^{(\log n)^{3}}}\left(\tilde{E}^{(\log n)^{3}}(\cdot \mid i)\right)-\Theta_{t^{(\log n)^{3}}}\right\| \leq \varepsilon
$$

for a $\Theta_{t^{(\log n)^{3}}} \in \mathcal{S}\left(H^{(\log n)^{3}}\right)$ which is independent of $j$.

We define $D_{j}:=L_{j-2}$ for $j \in\left\{3, \cdots, d^{m^{2}}+3\right\}$. For $i \in\left\{1, \cdots, n^{3}\right\}$ we define

$$
\tilde{D}_{i}^{(\log n)^{3}}:=\frac{1}{2} \sum_{j^{m} \in \tilde{S}_{i}^{(\log n)^{3}}} D_{j^{m}} .
$$


Here for $j^{m}=\left(j_{1}, \cdots, j_{m}\right)$ we set $D_{j^{m}}=D_{j_{1}} \otimes \cdots \otimes D_{j_{m}}$. Since $\sum_{i=1}^{n^{3}} \tilde{D}_{i}^{(\log n)^{3}}$ $=\frac{1}{2} \sum_{j^{m} \in\left\{1, \cdots, d^{m^{2}}+3\right\}^{m}} D_{j^{m}}=i d_{H^{m}},\left\{\tilde{D}_{i}^{(\log n)^{3}}: i \in\left\{1, \cdots, n^{3}\right\}\right\}$ is a valid set of decoding operators.

$$
\begin{aligned}
& \left(\tilde{E}^{(\log n)^{3}},\left\{\tilde{D}_{i}^{(\log n)^{3}}: i \in\left\{1, \cdots, n^{3}\right\}\right\}\right) \text { is a }\left((\log n)^{3}, n^{3}\right) \text { code which fulfills } \\
& \min _{t^{(\log n)^{3}} \in \theta^{(\log n)^{3}}} \frac{1}{n^{3}} \sum_{i=1}^{n^{3}} \operatorname{tr}\left(\check{W}_{t^{(\log n)^{3}}}\left(\tilde{E}^{(\log n)^{3}}(\cdot \mid i)\right) \tilde{D}_{i}^{(\log n)^{3}}\right) \geq 1-2^{-n^{1 / 16} \lambda} .
\end{aligned}
$$

v) The Secure Transmission of Both the Message and the Randomization Index

We choose an arbitrary positive $\delta$. Let

$$
J_{n}=\frac{1}{n}\left(\max _{U \rightarrow A \rightarrow\left\{B_{q}^{n}, Z_{t^{n}}: q, t_{n}\right\}} \inf _{B_{q} \in \operatorname{Conv}\left(\left(B_{t}\right)_{t \in \theta}\right)} \chi\left(p_{U} ; B_{q}^{\otimes n}\right)-\max _{t^{n} \in \theta^{n}} \chi\left(p_{U} ; Z_{t^{n}}\right)\right)-\delta .
$$

By the results of [20] if $n$ is sufficiently large there is a $\left(n, J_{n}\right)$ common randomness assisted quantum code $\left\{\left(\pi \circ E^{n},\left\{P_{\pi} D_{j}^{n} P_{\pi}^{T}: j \in\left\{1, \cdots, J_{n}\right\}\right\}\right): \pi \in \mathrm{S}_{n}\right\}$, a quantum state $\Theta_{t^{n}} \in \mathcal{S}\left(H^{n}\right)$ such that for all $t^{n} \in \theta^{n}$

$$
\frac{1}{n !} \frac{1}{J_{n}} \sum_{\pi \in S_{n}} \sum_{j=1}^{J_{n}} \operatorname{tr}\left(\check{W}_{t^{n}}\left(\pi\left(E^{n}(\cdot \mid j)\right)\right) P_{\pi} D_{\pi\left(t^{n}\right)} P_{\pi}^{T}\right) \geq 1-2^{-n^{1 / 16} \lambda},
$$

and for all $t^{n} \in \theta^{n}, j \in\left\{1, \cdots, J_{n}\right\}$ and all $\pi \in \mathrm{S}_{n}$

$$
\left\|\check{V}_{t^{n}}\left(\pi\left(E^{n}(\cdot \mid j)\right)\right)-P_{\pi} \Theta_{\pi\left(t^{n}\right)} P_{\pi}^{T}\right\|<2^{-\sqrt{n} \zeta}
$$

for a $\Theta_{t^{n}} \in \mathcal{S}\left(H^{n}\right)$ which is independent of $j$.

Using technique in [11] to reduce the amount of common randomness if $n$ is sufficiently large we can find a set $\left\{\pi_{1}, \cdots, \pi_{n^{3}}\right\} \subset \mathrm{S}_{n}$ such that

$$
\max _{t^{n} \in \theta^{n}} \frac{1}{n^{3}} \frac{1}{J_{n}} \sum_{i=1}^{n^{3}} \sum_{j=1}^{J_{n}} \operatorname{tr}\left(\check{W}_{t^{n}}\left(\pi\left(E^{n}(\cdot \mid j)\right)\right) P_{\pi_{i}} D_{\pi\left(t^{n}\right)} P_{\pi_{i}}^{T}\right) \geq 1-2 \cdot 2^{-n^{1 / 16} \lambda},
$$

and

$$
\left\|\check{V}_{t^{n}}\left(\pi_{i}\left(E^{n}(\cdot \mid j)\right)\right)-P_{\pi_{i}} \Theta_{\pi_{i}\left(t^{n}\right)} P_{\pi_{i}}^{T}\right\|<2^{-\sqrt{n} \zeta}
$$

Furthermore by the permutation-invariance of $p^{\prime}$ we also have $\Theta_{t^{n}}=P_{\pi} \Theta_{\pi\left(t^{n}\right)} P_{\pi}^{T}$ for all $\pi \in \mathrm{S}_{n}$.

Now we can construct a $\left((\log n)^{3}+n, n^{3} J_{n}\right)$ code $\left(E^{(\log n)^{3}+n},\left\{D_{i, j}^{(\log n)^{3}+n}: i=\right.\right.$ $\left.\left.1, \cdots, n^{3}, j=1, \cdots J_{n}\right\}\right)$ by

$$
E^{(\log n)^{3}+n}\left(a^{(\log n)^{3}+n} \mid i, j\right):=\tilde{E}^{(\log n)^{3}}\left(a^{(\log n)^{3}} \mid i\right) \cdot E^{n}\left(\pi_{i}\left(a^{n}\right) \mid j\right),
$$

for every $a^{(\log n)^{3}+n}=\left(a^{(\log n)^{3}}, a^{n}\right) \in \mathbf{U}^{(\log n)^{3}+n}$ and

$$
D_{i, j}^{(\log n)^{3}+n}:=\tilde{D}_{i}^{(\log n)^{3}} \otimes\left(P_{\pi_{i}} D_{j}^{n} P_{\pi_{i}}^{T}\right) .
$$


By 43 and 46 for every $t^{(\log n)^{3}+n}=\left(t^{(\log n)^{3}}, t^{n}\right) \in \theta^{(\log n)^{3}+n}$ we have

$$
\begin{aligned}
& \frac{1}{n^{3}} \frac{1}{J_{n}} \sum_{i=1}^{n^{3}} \sum_{j=1}^{J_{n}} \operatorname{tr}\left(\check{W}_{t^{(\log n)^{3}+n}}\left(E^{(\log n)^{3}+n}(\cdot \mid i, j)\right) D_{i, j}^{(\log n)^{3}+n}\right) \\
& =\frac{1}{n^{3}} \frac{1}{J_{n}} \sum_{i=1}^{n^{3}} \sum_{j=1}^{J_{n}} \operatorname{tr}\left(\left[\check{W}_{t^{(\log n)^{3}}}\left(\tilde{E}^{(\log n)^{3}}(\cdot \mid i)\right) \otimes\left(\check{W}_{t^{n}}\left(\pi_{i}\left(E^{n}(\cdot \mid j)\right)\right)\right)\right]\left[\tilde{D}_{i}^{(\log n)^{3}} \otimes\left(P_{\pi_{i}} D_{j}^{n} P_{\pi_{i}}^{T}\right)\right]\right) \\
& =\frac{1}{n^{3}} \sum_{i=1}^{n^{3}} \operatorname{tr}\left(\left[\check{W}_{t^{(\log n)^{3}}}\left(\tilde{E}^{(\log n)^{3}}(\cdot \mid i)\right) \tilde{D}_{i}^{(\log n)^{3}}\right] \otimes\left[\frac{1}{J_{n}} \sum_{j=1}^{J_{n}}\left(\check{W}_{t^{n}}\left(\pi_{i}\left(E^{n}(\cdot \mid j)\right)\right)\right) P_{\pi_{i}} D_{j}^{n} P_{\pi_{i}}^{T}\right]\right) \\
& =\frac{1}{n^{3}} \sum_{i=1}^{n^{3}}\left(\operatorname{tr}\left(\check{W}_{t^{(\log n)^{3}}}\left(\tilde{E}^{(\log n)^{3}}(\cdot \mid i)\right) \tilde{D}_{i}^{(\log n)^{3}}\right) \cdot \operatorname{tr}\left(\frac{1}{J_{n}} \sum_{j=1}^{J_{n}}\left(\check{W}_{t^{n}}\left(\pi_{i}\left(E^{n}(\cdot \mid j)\right)\right)\right) P_{\pi_{i}} D_{j}^{n} P_{\pi_{i}}^{T}\right)\right) \\
& \geq 1-\frac{1}{n^{1 / 16}} 2^{\lambda}-2 \cdot 2^{-n^{1 / 16} \lambda} \\
& \geq 1-\varepsilon
\end{aligned}
$$

for any positive $\varepsilon$.

By 422 and 477 for every $t^{(\log n)^{3}+n}=\left(t^{(\log n)^{3}}, t^{n}\right) \in \theta^{(\log n)^{3}+n}$ and $i \in$ $\left\{1, \cdots, n^{3}\right\}$ and $j \in\left\{1, \cdots, J_{n}\right\}$ we have

$$
\begin{aligned}
& \left\|\check{V}_{t^{(\log n)^{3}+n}}\left(E^{(\log n)^{3}+n}(\cdot \mid i, j)\right)-\Theta_{t^{(\log n)^{3}}} \otimes \Theta_{t^{n}}\right\| \\
& =\left\|\check{V}_{t^{(\log n)^{3}}}\left(\tilde{E}^{(\log n)^{3}}(\cdot \mid i)\right) \otimes \check{V}_{t^{n}}\left(\pi\left(E^{n}(\cdot \mid j)\right)\right)-\Theta_{t^{(\log n)^{3}}} \otimes \Theta_{t^{n}}\right\| \\
& <\frac{1}{\sqrt{n}} 2^{\zeta}+2^{-\sqrt{n} \zeta} .
\end{aligned}
$$

Let $R_{n^{3}}$ be the uniform distribution on $\left\{1, \cdots, n^{3}\right\}$. We define a random variable $R_{n^{3}, \text { uni }}$ on the set $\left\{1, \cdots, n^{3}\right\} \times\left\{1, \cdots, R_{n}\right\}$ by $R_{n^{3}, \text { uni }}:=R_{n^{3}} \times R_{u n i}$. Applying Lemma 3.4 we obtain

$$
\begin{aligned}
& \max _{t^{(\log n)^{3}+n} \in \theta^{(\log n)^{3}+n}} \chi\left(R_{n^{3}, J_{n}}, Z_{t^{(\log n)^{3}+n}}\right) \\
& \leq \max _{t^{(\log n)^{3}} \in \theta^{(\log n)^{3}}} \chi\left(R_{n^{3}}, Z_{t^{(\log n)^{3}+n}}\right) \\
& +\frac{1}{n^{3}} \sum_{i=1}^{n^{3}} \max _{t^{n} \in \theta^{n}} \chi\left(R_{u n i}, \check{V}_{t^{(\log n)^{3}}}\left(\tilde{E}^{(\log n)^{3}}(\cdot \mid i)\right) \otimes Z_{t^{n}, \pi_{i}}\right) \\
& =\max _{t^{(\log n)^{3} \in \theta^{(\log n)^{3}}}}\left(S\left(\frac{1}{n^{3}} \frac{1}{J_{n}} \sum_{i=1}^{n^{3}} \sum_{j=1}^{J_{n}} \check{V}_{t^{(\log n)^{3}+n}}\left(E^{(\log n)^{3}+n}(\cdot \mid i, j)\right)\right)\right. \\
& \left.-\frac{1}{n^{3}} \sum_{i=1}^{n^{3}} S\left(\frac{1}{J_{n}} \sum_{j=1}^{J_{n}} \check{V}_{t^{(\log n)^{3}+n}}\left(E^{(\log n)^{3}+n}(\cdot \mid i, j)\right)\right)\right)
\end{aligned}
$$




$$
\begin{aligned}
& +\max _{t^{n} \in \theta^{n}} \frac{1}{n^{3}} \sum_{i=1}^{n^{3}}\left(S\left(\frac{1}{n^{3}} \frac{1}{J_{n}} \sum_{i=1}^{n^{3}} \sum_{j=1}^{J_{n}} \check{V}_{t^{(\log n)^{3}}}\left(\tilde{E}^{(\log n)^{3}}(\cdot \mid i)\right) \otimes\left(\check{V}_{t^{n}}\left(\pi_{i}\left(E^{n}(\cdot \mid j)\right)\right)\right)\right)\right. \\
& \left.-\frac{1}{J_{n}} \sum_{j=1}^{J_{n}} S\left(\check{V}_{t^{(\log n)^{3}}}\left(\tilde{E}^{(\log n)^{3}}(\cdot \mid i)\right) \otimes\left(\check{V}_{t^{n}}\left(\pi_{i}\left(E^{n}(\cdot \mid j)\right)\right)\right)\right)\right) \\
& \leq \max _{t^{(\log n)^{3}} \in \theta^{(\log n)^{3}}}\left(\left|S\left(\frac{1}{n^{3}} \frac{1}{J_{n}} \sum_{i=1}^{n^{3}} \sum_{j=1}^{J_{n}} \check{V}_{t^{(\log n)^{3}+n}}\left(E^{(\log n)^{3}+n}(\cdot \mid i, j)\right)\right)-\Theta_{t^{(\log n)^{3}}} \otimes \Theta_{t^{n}}\right|\right. \\
& \left.+\left|\Theta_{t^{(\log n)^{3}}} \otimes \Theta_{t^{n}}-\frac{1}{n^{3}} \sum_{i=1}^{n^{3}} S\left(\frac{1}{J_{n}} \sum_{j=1}^{J_{n}} \check{V}_{t^{(\log n)^{3}+n}}\left(E^{(\log n)^{3}+n}(\cdot \mid i, j)\right)\right)\right|\right) \\
& +\max _{t^{n} \in \theta^{n}} \frac{1}{n^{3}}\left(\mid S\left(\frac{1}{J_{n}} \sum_{j=1}^{J_{n}} \check{V}_{t^{(\log n)^{3}}}\left(\tilde{E}^{(\log n)^{3}}(\cdot \mid i)\right) \otimes\left(\check{V}_{t^{n}}\left(\pi_{i}\left(E^{n}(\cdot \mid j)\right)\right)\right)-\check{V}_{t^{(\log n)^{3}}}\left(\tilde{E}^{(\log n)^{3}}(\cdot \mid i)\right) \otimes \Theta_{t^{n}} \mid\right.\right. \\
& \left.+\left|\check{V}_{t^{(\log n)^{3}}}\left(\tilde{E}^{(\log n)^{3}}(\cdot \mid i)\right) \otimes \Theta_{t^{n}}-\frac{1}{J_{n}} \sum_{j=1}^{J_{n}} S\left(\check{V}_{t^{(\log n)^{3}}}\left(\tilde{E}^{(\log n)^{3}}(\cdot \mid i)\right) \otimes\left(\check{V}_{t^{n}}\left(\pi_{i}\left(E^{n}(\cdot \mid j)\right)\right)\right)\right)\right|\right) \\
& \leq\left(\frac{1}{\sqrt{n}} 2^{\zeta}+2^{-\sqrt{n} \zeta}\right) \log \left(d^{(\log n)^{3}}-1\right)+h\left(\frac{1}{\sqrt{n}} 2^{\zeta}+2^{-\sqrt{n} \zeta}\right) \\
& +2^{-\sqrt{n} \zeta} \log \left(d^{n}-1\right)+h\left(2^{-\sqrt{n} \zeta}\right) \\
& \leq \varepsilon
\end{aligned}
$$

for any positive $\varepsilon$. Here $Z_{i, t^{n}}$ is the resulting quantum state at $\check{V}_{t^{n}}$ after $i \in$ $\left\{1, \cdots, n^{3}\right\}$ has been sent with $E^{(\log n)^{3}}$.

For any positive $\delta$, if $n$ is large enough we have $\frac{1}{n} \log J_{n}-\frac{1}{(\log n)^{3}+n} \log J_{n} \leq \delta$. Thus the secrecy rate of $\left\{\left(W_{t}, V_{t}\right): t \in \theta\right\}$ to transmission of both the message and the randomization index is large than

$$
\frac{1}{n} \max _{U \rightarrow A \rightarrow\left\{B_{q}, Z_{t}: q, t\right\}}\left(\inf _{B_{q} \in \operatorname{Conv}\left(\left(B_{t}\right)_{t \in \theta}\right)} \chi\left(p_{U} ; B_{q}^{\otimes n}\right)-\max _{t^{n} \in \theta^{n}} \chi\left(p_{U} ; Z_{t^{n}}\right)\right)-2 \delta .
$$

\section{Further Notice on Code Concepts and Appli- cations}

\subsection{Communication With Resources}

In our previous papers [11] and [12] we determined the randomness assisted secrecy capacities of arbitrarily varying classical-quantum wiretap channels. 


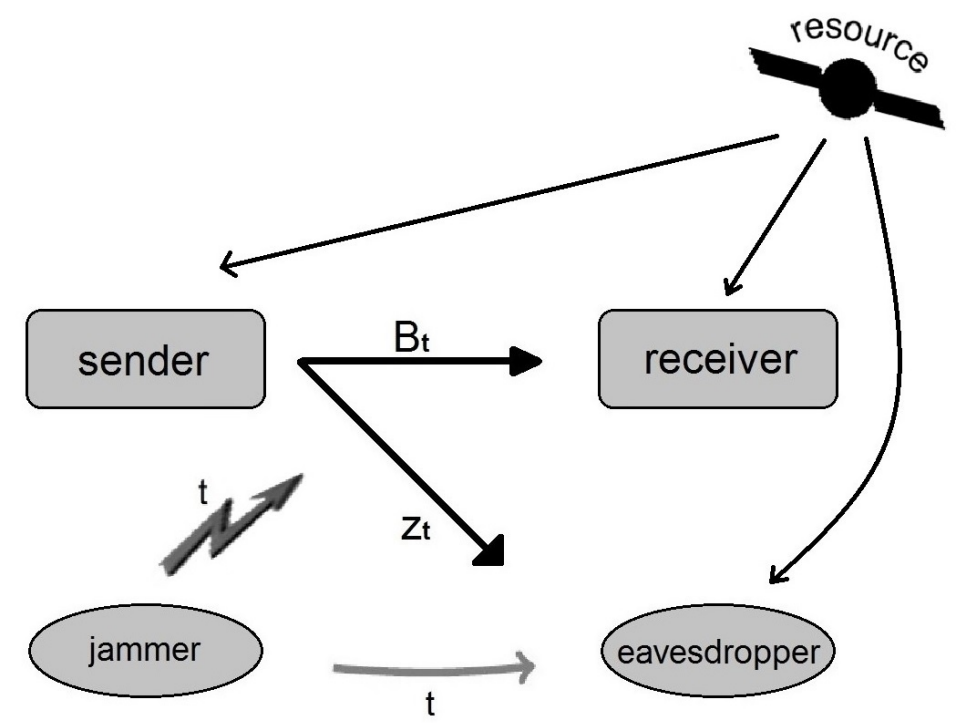

Figure 2: Arbitrarily varying classical-quantum wiretap channel with assistance by shared randomness that is not known by the jammer

In [11] we gave an example when the deterministic capacity of an arbitrarily varying classical-quantum wiretap channel is not equal to its randomnessassisted capacity. Thus having resources is very helpful for achieving a positive secrecy capacity. For the proofs in [11] and [12] we did not allow the jammer to have access to the shared randomness.

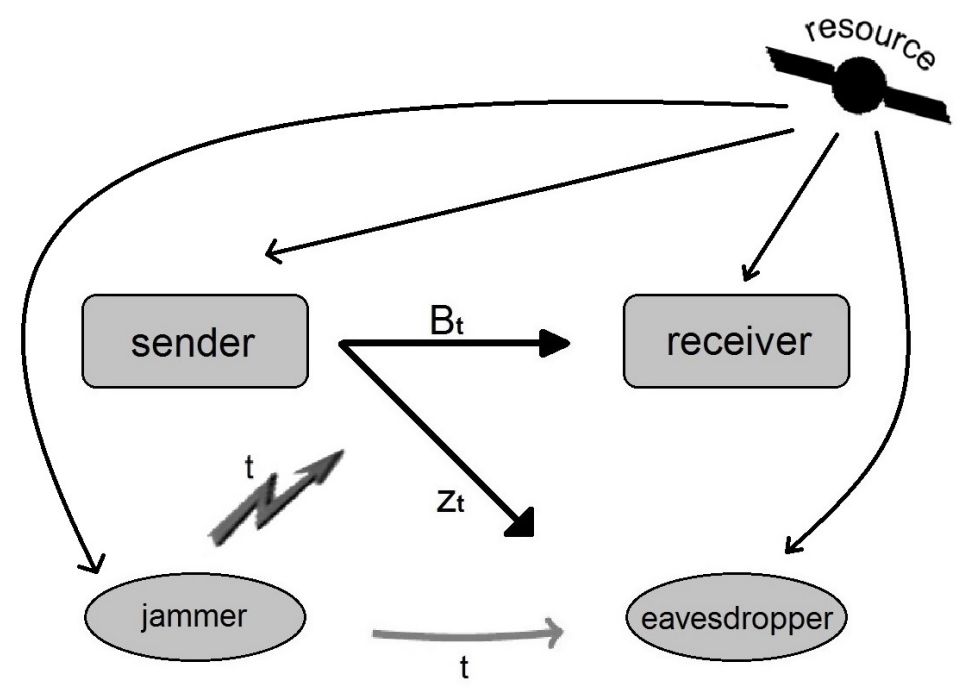

Figure 3: Arbitrarily varying classical-quantum wiretap channel with assistance by shared randomness that is known by the jammer 
Now we consider the case when the shared randomness is not secure, i.e. when the jammer can have access to the shared randomness.

Corollary 4.1. Let $\left\{\left(W_{t}, V_{t}\right): t \in \theta\right\}$ be an arbitrarily varying classical-quantum wiretap channel. We have

$$
C_{s}\left(\left\{\left(W_{t}, V_{t}\right): t \in \theta\right\}\right)=C_{s}\left(\left\{\left(W_{t}, V_{t}\right): t \in \theta\right\} ; r_{n s}\right)
$$

Proof. Let $\mathcal{C}=\left(E,\left\{D_{j}^{n}: j=1, \cdots J_{n}\right\}\right)$ be an $\left(n, J_{n}\right)$ code such

$$
\max _{t^{n} \in \theta^{n}} P_{e}\left(\mathcal{C}, t^{n}\right)<\epsilon,
$$

and

$$
\max _{t^{n} \in \theta^{n}} \chi\left(R_{u n i} ; Z_{t^{n}}\right)<\zeta .
$$

We define a $G^{\prime}$ such that $G^{\prime}(\{\mathcal{C}\})=1$, it holds

$$
\begin{gathered}
\int_{\Lambda} \max _{t^{n} \in \theta^{n}} P_{e}\left(\mathcal{C}^{\gamma}, t^{n}\right) d G^{\prime}(\gamma)<\epsilon, \\
\int_{\Lambda} \max _{t^{n} \in \theta^{n}} \chi\left(R_{u n i}, Z_{\mathcal{C}^{\gamma}, t^{n}}\right) d G^{\prime}(\gamma)<\zeta .
\end{gathered}
$$

Thus every achievable secrecy rate for $\left\{\left(W_{t}, V_{t}\right): t \in \theta\right\}$ is also an achievable secrecy rate for $\left\{\left(W_{t}, V_{t}\right): t \in \theta\right\}$ under non-secure randomness assisted coding.

Now we assume that there is a $G^{\prime \prime}$ such that

$$
\begin{gathered}
\int_{\Lambda} \max _{t^{n} \in \theta^{n}} P_{e}\left(\mathcal{C}^{\gamma}, t^{n}\right) d G^{\prime \prime}(\gamma)<\epsilon, \\
\int_{\Lambda} \max _{t^{n} \in \theta^{n}} \chi\left(R_{u n i}, Z_{\mathcal{C}^{\gamma}, t^{n}}\right) d G^{\prime \prime}(\gamma)<\zeta .
\end{gathered}
$$

Then for any $s^{n} \in \theta^{n}$ we have

$$
\begin{gathered}
\int_{\Lambda} P_{e}\left(\mathcal{C}^{\gamma}, s^{n}\right) d G^{\prime \prime}(\gamma) \leq \int_{\Lambda} \max _{t^{n} \in \theta^{n}} P_{e}\left(\mathcal{C}^{\gamma}, t^{n}\right) d G^{\prime \prime}(\gamma)<\epsilon, \\
\int_{\Lambda} \chi\left(R_{u n i}, Z_{\mathcal{C}^{\gamma}, s^{n}}\right) d G^{\prime \prime}(\gamma) \leq \int_{\Lambda} \max _{t^{n} \in \theta^{n}} \chi\left(R_{u n i}, Z_{\mathcal{C}^{\gamma}, t^{n}}\right) d G^{\prime \prime}(\gamma)<\zeta .
\end{gathered}
$$

Thus every achievable secrecy rate for $\left\{\left(W_{t}, V_{t}\right): t \in \theta\right\}$ under non-secure randomness assisted coding is also an achievable secrecy rate for $\left\{\left(W_{t}, V_{t}\right): t \in\right.$ $\theta\}$ under randomness assisted coding.

Therefore,

$$
C_{s}\left(\left\{\left(W_{t}, V_{t}\right): t \in \theta\right\}\right) \leq C_{s}\left(\left\{\left(W_{t}, V_{t}\right): t \in \theta\right\} ; r_{n s}\right) \leq C_{s}\left(\left\{\left(W_{t}, V_{t}\right): t \in \theta\right\} ; r\right) .
$$


At first let us assume that $\left\{W_{t}: t \in \theta\right\}$ is not symmetrizable. By [11] when $\left\{W_{t}: t \in \theta\right\}$ is not symmetrizable it holds $C_{s}\left(\left\{\left(W_{t}, V_{t}\right): t \in \theta\right\}\right)=$ $C_{s}\left(\left\{\left(W_{t}, V_{t}\right): t \in \theta\right\} ; r\right)$. Thus when $\left\{W_{t}: t \in \theta\right\}$ is not symmetrizable we have

$$
C_{s}\left(\left\{\left(W_{t}, V_{t}\right): t \in \theta\right\}\right)=C_{s}\left(\left\{\left(W_{t}, V_{t}\right): t \in \theta\right\} ; r_{n s}\right) .
$$

Now let us assume that $\left\{W_{t}: t \in \theta\right\}$ is symmetrizable. When $\left\{W_{t}: t \in \theta\right\}$ is symmetrizable and $J_{n}>1$ hold then by [11] for any $\left(n, J_{n}\right)$ code $\mathcal{C}$ there is are $t^{n} \in \theta^{n}$ and a positive $c$ such that

$$
P_{e}\left(\mathcal{C}, t^{n}\right)>c
$$

Thus when $\left\{W_{t}: t \in \theta\right\}$ is symmetrizable for any $G$ we have

$$
\int_{\Lambda} \max _{t^{n} \in \theta^{n}} P_{e}\left(\mathcal{C}^{\gamma}, t^{n}\right) d G(\gamma)>c
$$

which implies we can only have $\int_{\Lambda} \max _{t^{n} \in \theta^{n}} P_{e}\left(\mathcal{C}^{\gamma}, t^{n}\right) d G(\gamma)<c$ when $J_{n}$ is less or equal to 1 . This means

$$
C_{s}\left(\left\{\left(W_{t}, V_{t}\right): t \in \theta\right\} ; r_{n s}\right)=\log 1=0 .
$$

By [11] when $\left\{W_{t}: t \in \theta\right\}$ is symmetrizable it holds $C_{s}\left(\left\{\left(W_{t}, V_{t}\right): t \in \theta\right\}\right)=0$ and therefore when $\left\{W_{t}: t \in \theta\right\}$ is symmetrizable we have

$$
C_{s}\left(\left\{\left(W_{t}, V_{t}\right): t \in \theta\right\}\right)=C_{s}\left(\left\{\left(W_{t}, V_{t}\right): t \in \theta\right\} ; r_{n s}\right) .
$$

In 11 we showed that an arbitrarily varying classical-quantum channel with zero deterministic secrecy capacity allowed secure transmission if the sender and the legal receiver had the possibility to use shared randomness as long as the shared randomness was kept secret against the jammer. Corollary 4.1 shows that when the jammer is able have access to the outcomes of the shared random experiment we can only achieve the rate as when we do not use any shared randomness at all. This means the shared randomness will be completely useless when it is known by the jammer. 


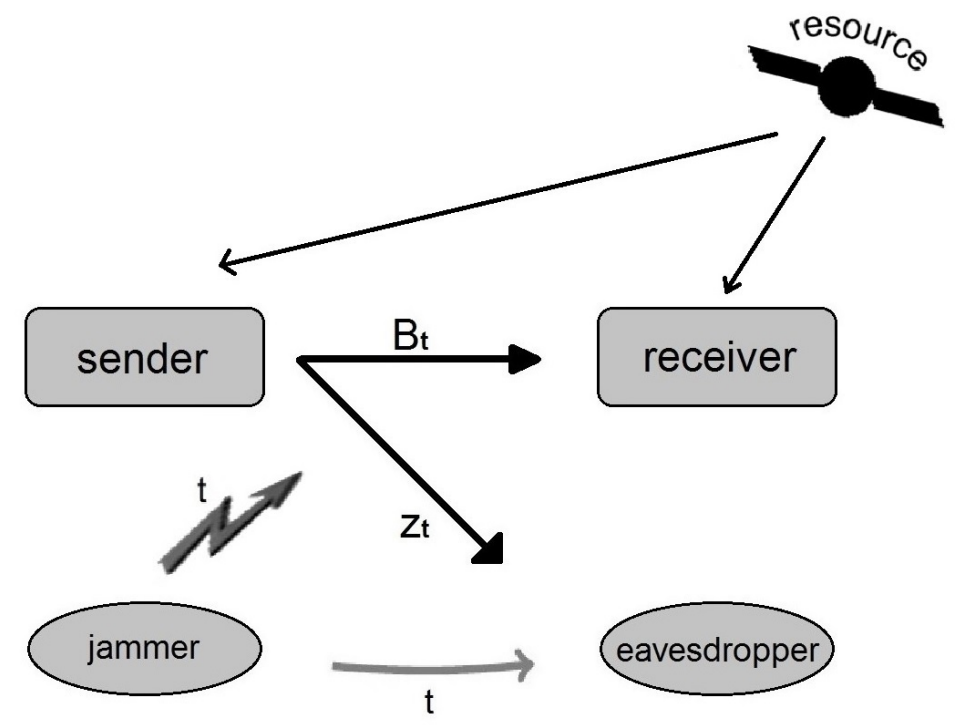

Figure 4: Arbitrarily varying classical-quantum wiretap channel with assistance by shared randomness that is not known by the eavesdropper

Applying Theorem 3.5 we can now determine the random assisted secrecy capacity with the strongest code concept for shared randomness, i.e., the randomness which is secure against both the jammer and eavesdropping.

Corollary 4.2. Let $\theta:=\{1, \cdots, T\}$ be a finite index set. Let $\left\{\left(W_{t}, V_{t}\right): t \in \theta\right\}$ be an arbitrarily varying classical-quantum wiretap channel.

When $\left\{W_{t}: t \in \theta\right\}$ is not symmetrizable, we have

$C_{k e y}\left(\left\{\left(W_{t}, V_{t}\right): t \in \theta\right\} ; g_{n}\right)$

$=\min \left(\lim _{n \rightarrow \infty} \frac{1}{n} \max _{U \rightarrow A \rightarrow\left\{B_{q}^{\otimes n}, Z_{t} n: q, t_{n}\right\}}\left(\inf _{B_{q} \in \operatorname{Conv}\left(\left(B_{t}\right)_{t \in \theta}\right)} \chi\left(p_{U} ; B_{q}^{\otimes n}\right)-\max _{t^{n} \in \theta^{n}} \chi\left(p_{U} ; Z_{t^{n}}\right)\right)+g_{n}\right.$,

$\left.\max _{U \rightarrow A \rightarrow\left\{B_{q}^{\otimes n}: q\right\}} \inf _{B_{q} \in \operatorname{Conv}\left(\left(B_{t}\right)_{t \in \theta}\right)} \chi\left(p_{U} ; B_{q}^{\otimes n}\right)\right)$.

Here we use the strong code concept.

Remark 4.3. When $g_{n}$ is positive and independent of $n$, 55) always holds and we do not have to assume that $\left\{W_{t}: t \in \theta\right\}$ is not symmetrizable.

Proof. We define $\Gamma_{n}^{\prime}:=\left\{1, \cdots,\left\lceil\frac{n^{3}}{\left|\Gamma_{n}\right|}\right\rceil\right\}$ it holds $n !>\left|\Gamma_{n}^{\prime} \times \Gamma_{n}\right| \geq n^{3}$. Notice that when $g_{n}$ is positive and independent of $n$ we always have have $n ! \geq 2^{n g_{n}} \geq n^{3}$ for sufficiently large $n$ and thus $\Gamma_{n}^{\prime}:=\{1\}$.

We fix a probability distribution $p \in P(\mathbf{A})$. Let

$$
J_{n}=\min \left(\left\lfloor 2^{n \min _{s \in \bar{\theta}} \chi\left(p ; B_{s}\right)-\log L_{n}+n g_{n}-2 n \mu}\right\rfloor,\left\lfloor 2^{n \min _{s \in \bar{\theta}} \chi\left(p ; B_{s}\right)-2 n \mu}\right\rfloor\right),
$$




$$
L_{n}=\max \left(\left\lceil 2^{\max _{t^{n}} \chi\left(p ; Z_{t} n\right)-n g_{n}+2 n \zeta}\right\rceil, 1\right) .
$$

and $p^{\prime}\left(x^{n}\right):=\left\{\begin{array}{ll}\frac{p^{n}\left(x^{n}\right)}{p^{n}\left(\mathrm{~T}_{p, \delta}^{n}\right)}, & \text { if } x^{n} \in \mathrm{T}_{p, \delta}^{n} ; \\ 0, & \text { else } .\end{array}\right.$ Let $X^{n}:=\left\{X_{j, l}: j \in\left\{1, \ldots, J_{n}\right\}, l \in\right.$ $\left.\left\{1, \ldots, L_{n}\right\}\right\}$ be a family of random variables taking value according to $p^{\prime}$.

It holds $J_{n} L_{n}<2^{n \min _{s \in \bar{\theta}} \chi\left(p ; B_{s}\right)}$ and $L^{n} 2^{g_{n}}>2^{\max _{t} n} \chi\left(p ; Z_{t}{ }^{n}\right)$. Similar to the proof of Theorem 1 in [11] and Theorem 3.1 in [12, with a positive probability there is a realization $\left\{x_{j, l}: j, l\right\}$ of $\left\{X_{j, l}: j, l\right\}$ and a set $\left\{\pi_{\gamma}: \gamma \in \Gamma_{n}^{\prime} \times \Gamma_{n}\right\}$ $\subset \mathrm{S}_{n}$ with following properties:

There exits a set of decoding operators $\left\{D_{j, l}: j=1, \cdots, J_{n}, l=1, \cdots, L_{n},\right\}$ such that for every $t^{n} \in \theta^{n} \epsilon>0, \zeta>0$, and sufficiently large $n$,

$$
1-\frac{1}{J_{n}} \frac{1}{L_{n}} \frac{1}{\left|\Gamma_{n}^{\prime} \times \Gamma_{n}\right|} \sum_{j=1}^{J_{n}} \sum_{j=1}^{L_{n}} \sum_{\gamma=1}^{\left|\Gamma_{n}^{\prime} \times \Gamma_{n}\right|} \operatorname{tr}\left(W_{t^{n}}\left(\pi_{\gamma}^{-1}\left(x_{j, l}\right)\right) P_{\pi_{\gamma}}^{\dagger} D_{j, l} P_{\pi_{\gamma}}\right)<\epsilon
$$

and

$$
\chi\left(R_{\text {uni }}, \frac{1}{J_{n}} \frac{1}{L_{n}} \frac{1}{\left|\Gamma_{n}^{\prime} \times \Gamma_{n}\right|} \sum_{j=1}^{J_{n}} \sum_{j=1}^{L_{n}} \sum_{\gamma=1}^{\left|\Gamma_{n}^{\prime} \times \Gamma_{n}\right|} V_{t^{n}}\left(\pi_{\gamma}^{-1}\left(x_{j, l}\right)\right)\right)<\zeta .
$$

When $\left|\Gamma_{n}^{\prime}\right|>1$ holds we use the strategy of Theorem 3.5 by build a two-part secure code word, the first part is used to send $\gamma^{\prime} \in \Gamma_{n}^{\prime}$, the second is used to transmit the message to the legal receiver.

Thus

$$
\begin{aligned}
& C_{k e y}\left(\left\{\left(W_{t}, V_{t}\right): t \in \theta\right\} ; g_{n}\right) \\
& \geq \min \left(\lim _{n \rightarrow \infty} \frac{1}{n} \max _{p}\left(\inf _{B_{q} \in \operatorname{Conv}\left(\left(B_{t}\right)_{t \in \theta}\right)} \chi\left(p ; B_{q}^{\otimes n}\right)-\max _{t^{n} \in \theta^{n}} \chi\left(p ; Z_{t^{n}}\right)\right)+g_{n},\right. \\
& \left.\max _{p} \inf _{B_{q} \in \operatorname{Conv}\left(\left(B_{t}\right)_{t \in \theta}\right)} \chi\left(p ; B_{q}^{\otimes n}\right)\right) .
\end{aligned}
$$

The achievability of $\lim _{n \rightarrow \infty} \frac{1}{n}\left(\min _{q} \chi\left(p_{U} ; B_{q}\right)-\max _{t^{n}} \chi\left(p_{U} ; Z_{t^{n}}\right)\right)+g_{n}$ and $\inf _{B_{q} \in \operatorname{Conv}\left(\left(B_{t}\right)_{t \in \theta}\right)} \chi\left(p_{U} ; B_{q}^{\otimes n}\right)$ is then shown via standard arguments.

Now we are going to prove the converse.

$$
C_{k e y}\left(\left\{\left(W_{t}, V_{t}\right): t \in \theta\right\} ; g_{n}\right) \leq \max _{U \rightarrow A \rightarrow\left\{B_{q}^{\otimes n}: q\right\}} \inf _{B_{q} \in \operatorname{Conv}\left(\left(B_{t}\right)_{t \in \theta}\right)} \chi\left(p_{U} ; B_{q}^{\otimes n}\right)
$$

holds trivially.

Let $\left(E^{\gamma,(n)},\left\{D_{j}^{\gamma,(n)}: j\right\}\right)$ be a sequence of $\left(n, J_{n}\right)$ code such that for every $t^{n} \in \theta^{n}$

$$
1-\frac{1}{J_{n}} \frac{1}{2^{n g_{n}}} \sum_{j=1}^{J_{n}} \sum_{\gamma=1}^{2^{n g_{n}}} \operatorname{tr}\left(W_{t^{n}}\left(E^{\gamma,(n)}(j)\right) D_{j}^{\gamma,(n)}\right)<\epsilon_{n}
$$

and

$$
\chi\left(R_{u n i}, \frac{1}{J_{n}} \frac{1}{2^{n g_{n}}} \sum_{j=1}^{J_{n}} \sum_{\gamma=1}^{2^{n g_{n}}} V_{t^{n}}\left(E^{\gamma,(n)}(j)\right)\right)<\zeta_{n}
$$


where $\lim _{n \rightarrow \infty} \epsilon_{n}=0$ and $\lim _{n \rightarrow \infty} \zeta_{n}=0$. It is known that for sufficiently large $n$ we have

$$
\log J_{n} \leq \frac{1}{2^{n g_{n}}} \sum_{\gamma=1}^{2^{n g_{n}}} \chi\left(R_{u n i}, B_{q}^{\gamma \otimes n}\right)-\chi\left(R_{u n i}, Z_{t^{n}}\right) .
$$

Let $\psi_{q}^{j, \gamma \otimes n}:=W_{q}^{\otimes n}\left(E^{\gamma,(n)}(j)\right)$. We denote $\tilde{B}_{q}^{j \otimes n}:=\left\{W_{q}^{\otimes n}\left(E^{\gamma,(n)}(j)\right): \gamma \in\right.$ $\left.\Gamma_{n}\right\}$ and $\tilde{B}_{q}^{\otimes n}:=\left\{\frac{1}{J_{n}} W_{q}^{\otimes n}\left(E^{\gamma,(n)}(j)\right): \gamma \in \Gamma_{n}\right\}$. Let $G_{u n i}$ be the uniformly distributed random variable with value in $\Gamma_{n}$.

We have

$$
\begin{aligned}
& \frac{1}{2^{n g_{n}}} \sum_{\gamma=1}^{2^{n g_{n}}} \chi\left(R_{u n i} ; B_{q}^{\gamma \otimes n}\right)-\chi\left(R_{\text {uni }} ; \frac{1}{2^{n g_{n}}} \sum_{\gamma=1}^{2^{n g_{n}}} B_{q}^{\gamma \otimes n}\right) \\
& =\frac{1}{2^{n g_{n}}} \sum_{\gamma=1}^{2^{n g_{n}}} S\left(\frac{1}{J_{n}} \sum_{j=1}^{J_{n}} \psi_{q}^{j, \gamma \otimes n}\right)-\frac{1}{2^{n g_{n}}} \frac{1}{J_{n}} \sum_{\gamma=1}^{2^{n g_{n}}} \sum_{j=1}^{J_{n}} S\left(\psi_{q}^{j, \gamma \otimes n}\right) \\
& -\left[S\left(\frac{1}{2^{n g_{n}}} \frac{1}{J_{n}} \sum_{\gamma=1}^{2^{n g_{n}}} \sum_{j=1}^{J_{n}} \psi_{q}^{j, \gamma \otimes n}\right)-\frac{1}{J_{n}} \sum_{j=1}^{J_{n}} S\left(\frac{1}{2^{n g_{n}}} \sum_{\gamma=1}^{2^{n g_{n}}} \psi_{q}^{j, \gamma \otimes n}\right)\right] \\
& =\frac{1}{2^{n g_{n}}} \sum_{\gamma=1}^{2^{n g_{n}}} S\left(\frac{1}{J_{n}} \sum_{j=1}^{J_{n}} \psi_{q}^{j, \gamma \otimes n}\right)-S\left(\frac{1}{2^{n g_{n}}} \frac{1}{J_{n}} \sum_{\gamma=1}^{2^{n g_{n}}} \sum_{j=1}^{J_{n}} \psi_{q}^{j, \gamma \otimes n}\right) \\
& -\left[\frac{1}{2^{n g_{n}}} \frac{1}{J_{n}} \sum_{\gamma=1}^{2^{n g_{n}}} \sum_{j=1}^{J_{n}} S\left(\psi_{q}^{j, \gamma \otimes n}\right)-\frac{1}{J_{n}} \sum_{j=1}^{J_{n}} S\left(\frac{1}{2^{n g_{n}}} \sum_{\gamma=1}^{2^{n g_{n}}} \psi_{q}^{j, \gamma \otimes n}\right)\right] \\
& =\frac{1}{J_{n}} \sum_{j=1}^{J_{n}} S\left(G_{u n i}, \tilde{B}_{q}^{j \otimes n}\right)-S\left(G_{u n i}, \tilde{B}_{q}^{\otimes n}\right) \\
& \leq \frac{1}{J_{n}} \sum_{j=1}^{J_{n}} S\left(G_{u n i}, \tilde{B}_{q}^{j \otimes n}\right) \\
& \leq \frac{1}{J_{n}} \sum_{j=1}^{J_{n}} H\left(G_{u n i}\right) \\
& =H\left(G_{u n i}\right) \\
& =n g_{n} .
\end{aligned}
$$

By (56), 57), and (58) we have

$$
\begin{aligned}
& C_{\text {key }}\left(\left\{\left(W_{t}, V_{t}\right): t \in \theta\right\} ; g_{n}\right) \\
& \leq \lim _{n \rightarrow \infty} \frac{1}{n} \max _{U \rightarrow A \rightarrow\left\{B_{q}^{\otimes n}, Z_{\left.t^{n}: q, t_{n}\right\}}\right.}\left(\inf _{B_{q} \in \operatorname{Conv}\left(\left(B_{t}\right)_{t \in \theta}\right)} \chi\left(p_{U} ; B_{q}^{\otimes n}\right)\right. \\
& \left.-\max _{t^{n} \in \theta^{n}} \chi\left(p_{U} ; Z_{t^{n}}\right)\right)+g_{n} .
\end{aligned}
$$




\subsection{Some Applications}

In this section we present some applications of our results in [1] and [12].

In 11] it has been shown that the deterministic secrecy capacity of an arbitrarily varying classical-quantum wiretap channel is in general not continuous. Now we deliver the sufficient and necessary conditions for the continuity of the capacity function of arbitrarily varying classical-quantum wiretap channels.

Corollary 4.4. For an arbitrarily varying classical-quantum channel $\left\{W_{t}: t \in\right.$ $\theta\}$ we define

$$
F\left(\left\{W_{t}: t\right\}\right):=\min _{\tau \in C(\theta \mid \mathbf{A})} \max _{a, a^{\prime}}\left\|\sum_{t \in \theta} \tau(t \mid a) W_{t}\left(a^{\prime}\right)-\sum_{t \in \theta} \tau\left(t \mid a^{\prime}\right) W_{t}(a)\right\|_{1},
$$

where $C(\theta \mid \mathbf{A})$ the set of parametrized distributions sets $\{\tau(\cdot \mid a): a \in \mathbf{A}\}$ on $\theta$. The statement $F\left(\left\{W_{t}: t\right\}\right)=0$ is equivalent to $\left\{W_{t}: t \in \theta\right\}$ being symmetrizable.

For an arbitrarily varying classical-quantum wiretap channel $\left\{\left(W_{t}, V_{t}\right): t \in\right.$ $\theta\}$, where $W_{t}: P(\mathbf{A}) \rightarrow \mathcal{S}(H)$ and $V_{t}: P(\mathbf{A}) \rightarrow \mathcal{S}\left(H^{\prime}\right)$, and a positive $\delta$ let $\mathrm{C}_{\delta}$ be the set of all arbitrarily varying classical-quantum wiretap channels $\left\{\left(W^{\prime}{ }_{t}, V^{\prime}{ }_{t}\right): t \in \theta\right\}$, where $W^{\prime}{ }_{t}: P(\mathbf{A}) \rightarrow \mathcal{S}(H)$ and $V^{\prime}{ }_{t}: P(\mathbf{A}) \rightarrow \mathcal{S}\left(H^{\prime}\right)$, such that

$$
\max _{a \in \mathbf{A}}\left\|W_{t}(a)-W^{\prime}{ }_{t}(a)\right\|_{1}<\delta
$$

and

$$
\max _{a \in \mathbf{A}}\left\|V_{t}(a)-V^{\prime}{ }_{t}(a)\right\|_{1}<\delta
$$

for all $t \in \theta$.

$C_{s}\left(\left\{\left(W_{t}, V_{t}\right): t\right\}\right)$, the deterministic secrecy capacity of arbitrarily varying classical-quantum wiretap channel is discontinuous at $\left\{\left(W_{t}, V_{t}\right): t \in \theta\right\}$ if and only if the following hold:

1) the secrecy capacity of $\left\{\left(W_{t}, V_{t}\right): t \in \theta\right\}$ under common randomness assisted quantum coding is positive;

2) $F\left(\left\{W_{t}: t\right\}\right)=0$ but for every positive $\delta$ there is a $\left\{\left(W^{\prime}{ }_{t}, V^{\prime}{ }_{t}\right): t \in \theta\right\} \in \mathrm{C}_{\delta}$ such that $F\left(\left\{W^{\prime}{ }_{t}: t\right\}\right)>0$.

Proof. At first we assume that the secrecy capacity of $\left\{\left(W_{t}, V_{t}\right): t \in \theta\right\}$ under common randomness assisted quantum coding is positive and $F\left(\left\{W_{t}: t\right\}\right)=$ 0 . We choose a positive $\epsilon$ such that $C_{s}\left(\left\{\left(W_{t}, V_{t}\right): t\right\} ; c r\right)-\epsilon:=C>0$. By Corollary 5.1 in [12] the secrecy capacity under common randomness assisted quantum coding is continuous. Thus there exist a positive $\delta$ such that the for all $\left\{\left(W^{\prime}{ }_{t}, V^{\prime}{ }_{t}\right): t \in \theta\right\} \in \mathrm{C}_{\delta}$ we have

$$
C_{s}\left(\left\{\left(W^{\prime}{ }_{t}, V^{\prime}{ }_{t}\right): t \in \theta\right\} ; c r\right) \geq C_{s}\left(\left\{\left(W_{t}, V_{t}\right): t\right\} ; c r\right)-\epsilon .
$$

Now we assume that there is a $\left\{\left(W^{\prime \prime}{ }_{t}, V^{\prime \prime}{ }_{t}\right): t \in \theta\right\} \in \mathrm{C}_{\delta}$ such that $F\left(\left\{W^{\prime \prime}{ }_{t}\right.\right.$ : $t\})>0$. This means that $\left\{W^{\prime \prime}{ }_{t}: t\right\}$ is not symmetrizable. By Theorem 1 in [11] it holds

$$
C_{s}\left(\left\{\left(W^{\prime \prime}{ }_{t}, V^{\prime \prime}{ }_{t}\right): t \in \theta\right\}\right)=C_{s}\left(\left\{\left(W^{\prime \prime}{ }_{t}, V^{\prime \prime}{ }_{t}\right): t\right\} ; c r\right) \geq C>0 .
$$


Since $F\left(\left\{W_{t}: t\right\}\right)=0,\left\{W_{t}: t\right\}$ is symmetrizable. By Theorem 1 in [1]

$$
C_{s}\left(\left\{\left(W_{t}, V_{t}\right): t \in \theta\right\}\right)=0 .
$$

Therefore the deterministic secrecy capacity is discontinuous at $\left\{\left(W_{t}, V_{t}\right): t \in\right.$ $\theta$ \} when 1) and 2) hold.

Now let us consider the case when the deterministic secrecy capacity is discontinuous at $\left\{\left(W_{t}, V_{t}\right): t \in \theta\right\}$.

We fix a $\tau \in C(\theta \mid \mathbf{A})$ and $a, a^{\prime} \in \mathbf{A}$. The map

$$
\left\{\left(W_{t}, V_{t}\right): t \in \theta\right\} \rightarrow\left\|\sum_{t \in \theta} \tau(t \mid a) W_{t}\left(a^{\prime}\right)-\sum_{t \in \theta} \tau\left(t \mid a^{\prime}\right) W_{t}(a)\right\|_{1}
$$

is continuous in the following sense: When $\left\|\sum_{t \in \theta} \tau(t \mid a) W_{t}\left(a^{\prime}\right)-\sum_{t \in \theta} \tau\left(t \mid a^{\prime}\right) W_{t}(a)\right\|_{1}$ $=C$ holds then for every positive $\delta$ and any $\left\{\left(W^{\prime}{ }_{t}, V^{\prime}{ }_{t}\right): t \in \theta\right\} \in \mathrm{C}_{\delta}$ we have

$$
\left|\left\|\sum_{t \in \theta} \tau(t \mid a) W^{\prime}{ }_{t}\left(a^{\prime}\right)-\sum_{t \in \theta} \tau\left(t \mid a^{\prime}\right) W^{\prime}{ }_{t}(a)\right\|_{1}-C\right| \leq 2 \delta .
$$

Thus if for a $\tau \in C(\theta \mid \mathbf{A})$ we have $\left\|\sum_{t \in \theta} \tau(t \mid a) W_{t}\left(a^{\prime}\right)-\sum_{t \in \theta} \tau\left(t \mid a^{\prime}\right) W_{t}(a)\right\|_{1}$ $=C>0$ for all $a, a^{\prime} \in \mathbf{A}$, we also have

$$
\left\|\sum_{t \in \theta} \tau(t \mid a) W^{\prime}{ }_{t}\left(a^{\prime}\right)-\sum_{t \in \theta} \tau\left(t \mid a^{\prime}\right) W^{\prime}{ }_{t}(a)\right\|_{1} \geq C-2 \delta .
$$

This means that when $F\left(\left\{W_{t}: t\right\}\right)>0$ holds we can find a positive $\delta$ such that $F\left(\left\{W^{\prime}{ }_{t}: t\right\}\right)>0$ holds for all $\left\{\left(W^{\prime}{ }_{t}, V^{\prime}{ }_{t}\right): t \in \theta\right\} \in \mathrm{C}_{\delta}$. By Theorem 1 in [11] it holds

$$
C_{s}\left(\left\{\left(W^{\prime}{ }_{t}, V^{\prime}{ }_{t}\right): t \in \theta\right\}\right)=C_{s}\left(\left\{\left(W^{\prime}{ }_{t}, V^{\prime}{ }_{t}\right): t\right\} ; c r\right) \geq C>0 .
$$

By Corollary 5.1 in [12] $C_{s}\left(\left\{\left(W^{\prime}{ }_{t}, V^{\prime}{ }_{t}\right): t\right\} ; c r\right)$ is continuous.

Therefore, when the deterministic secrecy capacity is discontinuous at $\left\{\left(W_{t}, V_{t}\right)\right.$ : $t \in \theta\}, F\left(\left\{W_{t}: t\right\}\right)$ cannot be positive.

We consider now that $F\left(\left\{W_{t}: t\right\}\right)=0$ holds. By Theorem 1 in [1]

$$
C_{s}\left(\left\{\left(W_{t}, V_{t}\right): t \in \theta\right\}=0 .\right.
$$

When for every $\left\{\left(W^{\prime}{ }_{t}, V^{\prime}{ }_{t}\right): t \in \theta\right\} \in \mathrm{C}_{\delta}$ we have $F\left(\left\{W^{\prime}{ }_{t}: t\right\}\right)=0$, then by Theorem 1 in 11$]$

$$
C_{s}\left(\left\{\left(W^{\prime}{ }_{t}, V^{\prime}{ }_{t}\right): t \in \theta\right\}\right)=0
$$

and the deterministic secrecy capacity is thus continuous at $\left\{\left(W_{t}, V_{t}\right): t \in \theta\right\}$.

Therefore, when the deterministic secrecy capacity is discontinuous at $\left\{\left(W_{t}, V_{t}\right)\right.$ : $t \in \theta\}$, for every positive $\delta$ there is a $\left\{\left(W^{\prime}{ }_{t}, V^{\prime}{ }_{t}\right): t \in \theta\right\} \in \mathrm{C}_{\delta}$ such that $F\left(\left\{W^{\prime}{ }_{t}: t\right\}\right)>0$.

When for every positive $\delta$ there is a $\left\{\left(W^{\prime}{ }_{t}, V^{\prime}{ }_{t}\right): t \in \theta\right\} \in \mathrm{C}_{\delta}$ such that $F\left(\left\{W^{\prime}{ }_{t}: t\right\}\right)>0$ and $C_{s}\left(\left\{\left(W_{t}, V_{t}\right): t \in \theta\right\}, c r\right)=0$ holds, then by Theorem 1 in [11] we have

$$
C_{s}\left(\left\{\left(W^{\prime}{ }_{t}, V^{\prime}{ }_{t}\right): t \in \theta\right\}\right)=C_{s}\left(\left\{\left(W^{\prime}{ }_{t}, V^{\prime}{ }_{t}\right): t \in \theta\right\}, c r\right),
$$


and the deterministic secrecy capacity is continuous at $\left\{\left(W_{t}, V_{t}\right): t \in \theta\right\}$.

Therefore, when the deterministic secrecy capacity is discontinuous at $\left\{\left(W_{t}, V_{t}\right)\right.$ : $t \in \theta\}, C_{s}\left(\left\{\left(W_{t}, V_{t}\right): t \in \theta\right\}, c r\right)$ must be positive.

Corollary 4.5. Let $\left\{\left(W_{t}, V_{t}\right): t \in \theta\right\}$ be an arbitrarily varying classical-quantum wiretap channel. When the secrecy capacity of $\left\{\left(W_{t}, V_{t}\right): t \in \theta\right\}$ is positive then there is a $\delta$ such that for all $\left\{\left(W^{\prime}{ }_{t}, V^{\prime}{ }_{t}\right): t \in \theta\right\} \in \mathrm{C}_{\delta}$ we have

$$
C_{s}\left(\left\{\left(W^{\prime}{ }_{t}, V^{\prime}{ }_{t}\right): t \in \theta\right\}\right)>0 \text {. }
$$

Proof. Suppose we have $C_{s}\left(\left\{\left(W_{t}, V_{t}\right): t \in \theta\right\}\right)>0$. Then $\left\{W_{t}: t \in \theta\right\}$ is not symmetrizable, which means that $F\left(\left\{W_{t}: t\right\}\right)$ is positive. In the proof of Corollary 4.4 we show that $F$ is continuous. Thus there is a positive $\delta^{\prime}$ such that $F\left(\left\{W^{\prime}{ }_{t}: t\right\}\right)>0$ for all $\left\{\left(W^{\prime}{ }_{t}, V^{\prime}{ }_{t}\right): t \in \theta\right\} \in \mathrm{C}_{\delta^{\prime}}$. When $\left\{W_{t}: t \in \theta\right\}$ is not symmetrizable then we have $C_{s}\left(\left\{\left(W_{t}, V_{t}\right): t \in \theta\right\}, c r\right)=C_{s}\left(\left\{\left(W_{t}, V_{t}\right): t \in \theta\right\}\right)$ $>0$. By Corollary 5.1 in [12], the secrecy capacity under common randomness assisted quantum coding is continuous. Thus there is a positive $\delta^{\prime \prime}$ such that $C_{s}\left(\left\{\left(W^{\prime}{ }_{t}, V^{\prime}{ }_{t}\right): t \in \theta\right\}, c r\right)>0$ for all $\left\{\left(W^{\prime}{ }_{t}, V^{\prime}{ }_{t}\right): t \in \theta\right\} \in \mathrm{C}_{\delta^{\prime \prime}}$. We define $\delta$ $:=\min \left(\delta^{\prime}, \delta^{\prime \prime}\right)$ and the Corollary is shown.

One of the properties of classical channels is that in the majority of cases, if we have a channel system where two sub-channels are used together, the capacity of this channel system is the sum of the two sub-channels' capacities. Particularly, a system consisting of two orthogonal classical channels, where both are "useless" in the sense that they both have zero capacity for message transmission, the capacity for message transmission of the whole system is zero as well $(" 0+0=0 ")$.

In contrast to the classical information theory, it is known that in quantum information theory, there are examples of two quantum channels, $W_{1}$ and $W_{2}$, with zero capacity, which allow perfect transmission if they are used together, i.e., the capacity of their product $W_{1} \otimes W_{2}$ is positive. This is due to the fact that there are different reasons why a quantum channel can have zero capacity. We call this phenomenon "super-activation" (" $0+0>0$ "). In [11 super-activation has been shown for arbitrarily varying classical-quantum wiretap channels. Now we deliver a complete characterization of super-activation for arbitrarily varying classical-quantum wiretap channels.

Corollary 4.6. Let $\left\{\left(W_{t}, V_{t}\right): t \in \theta\right\}$ and $\left\{\left(W^{\prime}{ }_{t}, V^{\prime}{ }_{t}\right): t \in \theta\right\}$ be two arbitrarily varying classical-quantum wiretap channels.

1) If $C_{s}\left(\left\{\left(W_{t}, V_{t}\right): t \in \theta\right\}\right)=C_{s}\left(\left\{\left(W^{\prime}{ }_{t}, V^{\prime}{ }_{t}\right): t \in \theta\right\}\right)=0$ then $C_{s}\left(\left\{W_{t} \otimes\right.\right.$ $\left.\left.W^{\prime}{ }_{t^{\prime}}, V_{t} \otimes V^{\prime}{ }_{t^{\prime}}: t, t^{\prime} \in \theta\right\}\right)$ is positive if and only if $\left\{W_{t} \otimes W^{\prime}{ }_{t^{\prime}}: t, t^{\prime} \in \theta\right\}$ is not symmetrizable and $C_{s}\left(\left\{W_{t} \otimes W_{t^{\prime}}^{\prime}, V_{t} \otimes V^{\prime}{ }_{t^{\prime}}: t, t^{\prime} \in \theta\right\}, c r\right)$ is positive.

2) If the secrecy capacity under common randomness assisted quantum coding shows no super-activation for $\left\{\left(W_{t}, V_{t}\right): t \in \theta\right\}$ and $\left\{\left(W^{\prime}{ }_{t}, V^{\prime}{ }_{t}\right): t \in \theta\right\}$ then the secrecy capacity can only then show super-activation for $\left\{\left(W_{t}, V_{t}\right): t \in \theta\right\}$ and $\left\{\left(W^{\prime}{ }_{t}, V^{\prime}{ }_{t}\right): t \in \theta\right\}$ if one of $\left\{\left(W_{t}, V_{t}\right): t \in \theta\right\}$ and $\left\{\left(W^{\prime}{ }_{t}, V^{\prime}{ }_{t}\right): t \in \theta\right\}$ has positive secrecy capacity under common randomness assisted quantum coding and a symmetrizable legal channel and while the other one has zero secrecy capacity under common randomness assisted quantum coding and a non-symmetrizable legal channel. 
Proof. By Theorem 1 in [1] $C_{s}\left(\left\{W_{t} \otimes W_{t^{\prime}}^{\prime}, V_{t} \otimes V_{t^{\prime}}^{\prime}: t, t^{\prime} \in \theta\right\}\right)$ is equal to $C_{s}\left(\left\{W_{t} \otimes W^{\prime}{ }_{t^{\prime}}, V_{t} \otimes V^{\prime}{ }_{t^{\prime}}: t, t^{\prime} \in \theta\right\}, c r\right)$ when $\left\{W_{t} \otimes W^{\prime}{ }_{t^{\prime}}: t, t^{\prime} \in \theta\right\}$ is not symmetrizable and to zero when $\left\{W_{t} \otimes W_{t^{\prime}}^{\prime}: t, t^{\prime} \in \theta\right\}$ is symmetrizable. Thus 1) holds.

When $\left\{W_{t}: t \in \theta\right\}$ and $\left\{W^{\prime}{ }_{t}: t \in \theta\right\}$ are both symmetrizable then there exists two parametrized set of distributions $\{\tau(\cdot \mid a): a \in \mathbf{A}\},\left\{\tau^{\prime}(\cdot \mid a): a \in \mathbf{A}\right\}$ on $\theta$ such that for all $a, a^{\prime} \in \mathbf{A}$, we have $\sum_{t \in \theta} \tau(t \mid a) W_{t}\left(a^{\prime}\right)=\sum_{t \in \theta} \tau(t \mid$ $\left.a^{\prime}\right) W_{t}(a), \sum_{t \in \theta} \tau^{\prime}(t \mid a) W^{\prime}{ }_{t}\left(a^{\prime}\right)=\sum_{t \in \theta} \tau ;\left(t \mid a^{\prime}\right) W^{\prime}{ }_{t}(a)$, We can set $\tau\left(\left(t, t^{\prime}\right) \mid\right.$ $\left.\left(a, a^{\prime}\right)\right):=\tau(t \mid a) \tau^{\prime}\left(t^{\prime} \mid a^{\prime}\right)$ and obtain

$\sum_{\left(t, t^{\prime}\right) \in \theta \times \theta} \tau\left(\left(t, t^{\prime}\right) \mid\left(a_{1}, a_{1}^{\prime}\right)\right) W_{t}\left(a_{2}\right) \otimes W_{t^{\prime}}^{\prime}\left(a_{2}^{\prime}\right)=\sum_{\left(t, t^{\prime}\right) \in \theta \times \theta} \tau\left(\left(t, t^{\prime}\right) \mid\left(a_{2}, a_{2}^{\prime}\right)\right) W_{t}\left(a_{1}\right) \otimes W^{\prime}{ }_{t^{\prime}}\left(a_{1}^{\prime}\right)$

for all $\left(a_{1}, a_{1}^{\prime}\right),\left(a_{2}, a_{2}^{\prime}\right) \in \mathbf{A} \times \mathbf{A}$, which means that $\left\{W_{t} \otimes W^{\prime}{ }_{t}: t, t^{\prime} \in \theta\right\}$ is symmetrizable and super-activation does not occur because of 1$)$.

When $\left\{W_{t}: t \in \theta\right\}$ and $\left\{W^{\prime}{ }_{t}: t \in \theta\right\}$ are both not symmetrizable then their secrecy capacities are equal to their secrecy capacities under common randomness assisted quantum coding. When $C_{s}\left(\left\{\left(W_{t}, V_{t}\right): t \in \theta\right\}, c r\right)=C_{s}\left(\left\{\left(W^{\prime}{ }_{t}, V^{\prime}{ }_{t}\right)\right.\right.$ : $t \in \theta\}, c r)=0$. Because of our assumption $C_{s}\left(\left\{W_{t} \otimes W^{\prime}{ }_{t^{\prime}}, V_{t} \otimes V^{\prime}{ }_{t^{\prime}}: t, t^{\prime} \in \theta\right\}, c r\right)$ $=0$. By 1), super-activation cannot occur.

When one of $\left\{W_{t}: t \in \theta\right\}$ and $\left\{W^{\prime}{ }_{t}: t \in \theta\right\}$, say $\left\{W_{t}: t \in \theta\right\}$ is not symmetrizable while the other one is symmetrizable, then $C_{s}\left(\left\{\left(W_{t}, V_{t}\right): t \in \theta\right\}\right)$ $=0$ indicate that $C_{s}\left(\left\{\left(W_{t}, V_{t}\right): t \in \theta\right\}, c r\right)=0$. When $C_{s}\left(\left\{\left(W_{t}^{\prime}, V^{\prime}{ }_{t}\right): t \in\right.\right.$ $\theta\}, c r)$ is also zero then by our assumption super-activation cannot occur. Thus 2) holds.

\section{Acknowledgment}

Supports by the Bundesministerium für Bildung und Forschung (BMBF) via Grant 16KIS0118K and 16KIS0117K, the German Research Council (DFG) via Grant 1129/1-1, the ERC via Advanced Grant IRQUAT, the Spanish MINECO via Project FIS2013-40627-P, and the Generalitat de CatalunyaCIRIT via Project 2014 SGR 966 are gratefully acknowledged.

\section{References}

[1] R. Ahlswede, A note on the existence of the weak capacity for channels with arbitrarily varying channel probability functions and its relation to Shannon's zero error capacity, The Annals of Mathematical Statistics, Vol. 41, No. 3, 1970.

[2] R. Ahlswede, Elimination of correlation in random codes for arbitrarily varying channels, Z. Wahrscheinlichkeitstheorie verw. Gebiete, Vol. 44, 159-175, 1978.

[3] R. Ahlswede, I. Bjelaković, H. Boche, and J. Nötzel, Quantum capacity under adversarial quantum noise: arbitrarily varying quantum channels, Comm. Math. Phys. A, Vol. 317, No. 1, 103-156, 2013. 
[4] R. Ahlswede and V. Blinovsky, Classical capacity of classical-quantum arbitrarily varying channels, IEEE Trans. Inform. Theory, Vol. 53, No. 2, 526-533, 2007.

[5] K. M. R. Audenaert, A sharp continuity estimate for the von Neumann entropy, J. Phys. A: Math. Theor., Vol. 40, 8127-8136, 2007.

[6] I. Bjelaković and H. Boche, Classical capacities of averaged and compound quantum channels. IEEE Trans. Inform. Theory, Vol. 57, No. 7, 3360-3374, 2009 .

[7] I. Bjelaković, H. Boche, G. Janßen, and J. Nötzel, Arbitrarily varying and compound classical-quantum channels and a note on quantum zeroerror capacities, Information Theory, Combinatorics, and Search Theory, in Memory of Rudolf Ahlswede, H. Aydinian, F. Cicalese, and C. Deppe eds., LNCS Vol.7777, 247-283, arXiv:1209.6325, 2012.

[8] D. Blackwell, L. Breiman, and A. J. Thomasian, The capacities of a certain channel classes under random coding, Ann. Math. Statist. Vol. 31, No. 3, $558-567,1960$.

[9] V. Blinovsky and M. Cai, arbitrarily classical-quantum varying wiretap channel, Information Theory, Combinatorics, and Search Theory, in Memory of Rudolf Ahlswede, H. Aydinian, F. Cicalese, and C. Deppe eds., LNCS Vol.7777, 234-246, 2013.

[10] M. Bloch and J. N. Laneman, On the secrecy capacity of arbitrary wiretap channels, Communication, Control, and Computing, Forty-Sixth Annual Allerton Conference Allerton House, UIUC, USA, 818-825, 2008

[11] H. Boche, M. Cai, C. Deppe, and J. Nötzel, Classical-quantum arbitrarily varying wiretap channel - Ahlswede Dichotomy - positivity - resources super activation, Quantum Information Processing, Vol. 15, No. 11, 4853489, arXiv:1307.8007, 2016.

[12] H. Boche, M. Cai, C. Deppe, and J. Nötzel, Classical-quantum arbitrarily varying wiretap channel: Common randomness assisted code and continuity, Quantum Information Processing, Vol. 16, No. 1, 1-48, 2016.

[13] H. Boche and J. Nötzel, Arbitrarily small amounts of correlation for arbitrarily varying quantum channel, J. Math. Phys., Vol. 54, Issue 11, arXiv $1301.6063,2013$.

[14] N. Cai, A. Winter, and R. W. Yeung, Quantum privacy and quantum wiretap channels, Problems of Information Transmission, Vol. 40, No. 4, 318-336, 2004.

[15] I. Csiszár and P. Narayan, The capacity of the arbitrarily varying channel revisited: positivity, constraints, IEEE Trans. Inform. Theory, Vol. 34, No. 2, 181-193, 1988.

[16] I. Devetak, The private classical information capacity and quantum information capacity of a quantum channel, IEEE Trans. Inform. Theory, Vol. 51, No. 1, 44-55, 2005. 
[17] T. Ericson, Exponential error bounds for random codes in the arbitrarily varying channel, IEEE Trans. Inform. Theory, Vol. 31, No. 1, 42-48, 1985.

[18] M. Fannes, A continuity property of the entropy density for spin lattice systems, Communications in Mathematical Physics, Vol. 31. 291-294, 1973.

[19] A. S. Holevo, The capacity of quantum channel with general signal states, IEEE Trans. Inform. Theory, Vol. 44, 269-273, 1998.

[20] J. Nötzel, M. Wiese, and H. Boche, The Arbitrarily Varying Wiretap Channel - Secret Randomness, Stability and Super-Activation, IEEE Trans. Inform. Theory, Vol. 62, No. 6, 3504-3531, arXiv:1501.07439, 2016.

[21] T. Ogawa and H. Nagaoka, Making good codes for classical-quantum channel coding via quantum hypothesis testing, IEEE Trans. Inform. Theory, Vol. 53, No. 6, 2261-2266, 2007.

[22] B. Schumacher and M. A. Nielsen, Quantum data processing and error correction, Phys. Rev. A, Vol. 54, 2629, 1996.

[23] B. Schumacher and M. D. Westmoreland, Sending classical information via noisy quantum channels, Phys. Rev., Vol. 56, 131-138, 1997.

[24] M. Wiese, J. Nötzel, and H. Boche, The arbitrarily varying wiretap channel-deterministic and correlated random coding capacities under the strong secrecy criterion, IEEE Trans. Inform. Theory, Vol. 62, No. 7, 3844 - 3862, arXiv:1410.8078, 2016.

[25] M. Wilde, Quantum Information Theory, Cambridge University Press, 2013.

[26] A. Winter, Coding theorem and strong converse for quantum channels, IEEE Trans. Inform. Theory, Vol. 45, No. 7, 2481-2485, 1999.

[27] A. D. Wyner, The wire-tap channel, Bell System Technical Journal, Vol. 54, No. 8, 1355-1387, 1975. 